Ocean Engineering

January 2018, Volume 148 Pages 361-375

http://dx.doi.org/10.1016/i.oceaneng.2017.11.041

http://archimer.ifremer.fr/doc/00412/52329/

(c) 2017 Elsevier Ltd. All rights reserved.

\title{
Experimental study of the bubble sweep-down phenomenon on three bow designs
}

\author{
Mallat Bachar ${ }^{1}$, Germain Grégory ${ }^{1,{ }^{*}}$, Gaurier Benoit ${ }^{1}$, Druault Philippe ${ }^{2}$, Billard Jean-Yves ${ }^{3}$
}

1 IFREMER, Marine Structure Laboratory, 150 Quai Gambetta, 62321 Boulogne-sur-Mer, France

${ }^{2}$ Sorbonne Universités, UPMC Univ Paris 06, CNRS, UMR 7190, Institut Jean Le Rond d'Alembert, F75005 Paris, France

${ }^{3}$ IRENAV, Ecole Navale, 29240 Brest, France

* Corresponding author : Grégory Germain, email address : ggermain@ifremer.fr

\begin{abstract}
:
The bubble sweep-down phenomenon around the oceanographic research vessels generates acoustic perturbations. A specific experimental protocol has been developed in a wave and circulating tank to study this phenomenon. This protocol is used to carry out trials on three different ship models in order to study the influence of the bow geometry on the bubble generation. For different test configurations, bubble clouds are described and compared in terms of area, maximal depth and vertical velocity to highlight bubble cloud dynamics surrounding the three ship models. The relation between the hydrodynamic flow field and the bubble generation is studied by means of Particle Image Velocimetry (PIV) measurements to study the phenomenon by the use of phase averaged velocity fields. The overall results enable us to characterize the bubble sweep-down phenomenon from the air bubble generation and propagation to the frequency of occurrence and the clouds behaviour.
\end{abstract}

\section{Highlights}

- Air entrainment is studied on three kind of ship bow geometry. Bow geometry effects on depth, area and velocity bubble cloud characteristics are determined. PIV measurements and POD analysis allow to study the air bubble generation.

Keywords : Bubble sweep-down, Experimental trials, Bow geometry, Bubble cloud dynamics, PIV 


\section{List of symbols}

$L_{p p} \quad$ Length between perpendiculars $(m)$

$C_{B} \quad$ Block coefficient

$B \quad$ Beam $(m)$

$D \quad$ Draft $(m)$

$\lambda \quad$ Wave length $(m)$

$H \quad$ Wave height $(m)$

$\mathrm{Fr} \quad$ Froude number

Re Reynolds number

We Webber number

$g \quad$ Gravitational acceleration $\left(m . s^{-2}\right)$

$U \quad$ Current velocity $\left(m . s^{-1}\right)$

$\nu \quad$ Kinematic viscosity $\left(m^{2} . s^{-1}\right)$

$\sigma \quad$ Surface tension $\left(N . m^{-1}\right)$

$\rho \quad$ Fluid density $\left(g \cdot \mathrm{m}^{-3}\right)$

$f_{0} \quad$ Wave and ship motions frequency $(H z)$

$f \quad$ Occurrence frequency of bubble clouds $(\mathrm{Hz})$

$A_{0} \quad \mathrm{D}^{*} 0.2^{*} \operatorname{Lpp}\left(m^{2}\right)$

$Z_{0} \quad$ Model draft $(\mathrm{mm})$

$A_{\text {cloud }}$ Bubble cloud area at model scale $\left(\mathrm{mm}^{2}\right)$

$Z_{\max } \quad$ Bubble cloud depth at model scale $(\mathrm{mm})$

$W_{\text {cloud }}$ Bubble cloud vertical velocity at model scale $\left(m . s^{-1}\right)$

A Bubble cloud area at full scale $\left(\mathrm{m}^{2}\right)$

$Z \quad$ Bubble cloud depth at full scale $(m)$

$W \quad$ Bubble cloud vertical velocity at full scale $\left(m . s^{-1}\right)$

$T \quad$ Wave and ship motions period $(s)$

$N_{t} \quad$ Number of PIV snapshots

$W_{\text {pod }} \quad$ Vertical velocity component of the flow after the POD application $\left(m . s^{-1}\right)$

$X_{1} \quad$ A point near the bow

$X_{2} \quad$ A point far away from the bow

$w_{1} \quad$ Vertical velocity component of the flow at the point $X_{1}\left(m . s^{-1}\right)$

$w_{2} \quad$ Vertical velocity component of the flow at the point $X_{2}\left(m . s^{-1}\right)$

$d \omega \quad$ Velocity difference $w_{1}-w_{2}\left(m . s^{-1}\right)$ 


\title{
Abbreviations
}

\author{
PP Pourquoi pas? \\ IB Inverted Bow \\ TB Thin Bow \\ CAD Computer Aided Design \\ PIV Particle Image Velocimetry \\ POD Proper Orthogonal Decomposition
}

\section{${ }_{5} 1$ Introduction}

The bubble sweep-down phenomenon is a widely well known phenomenon even if it is not well understood. On many specialized vessels, such as oceanographic survey and research vessels, bubble-sweep down can significantly degrade the effectiveness of transducer performance. Even if the use of the acoustic technique has demonstrated its potential for the water column and the sea-bed characterization [23], it remains challenging for ship designers to select the optimal hull shape and sonar location to avoid the phenomenon. This phenomenon is divided into two important events [5]. The first one is the generation of air bubbles induced by a perturbation at the free surface. The second one is the entrainment of these bubbles by a path backwards along the ship hull and under the transducers which disrupt the acoustic signals and may result in a considerable reduction of the ships productivity [7].

The tools for the study of this phenomenon are therefore limited. The main difficulties come from the scale differences between the bubble generation, governed by the surface tension, and the overall flow around the ship. Experimental studies have been carried out by Waniewski [24] and Tavakolinejad [22] to study the air entrainment by the bow waves, but they do not take into account the sea state which is a significant parameter for the bubble sweep-down phenomenon.

Bonmarin [1] and Ducan [12, 13] have described the breaking waves dynamics in order to develop theoretical models. Many other experimental studies (Lamarre[17] and Deane and Stockes [5]) have been carried out to measure the void fraction and bubble size distribution in breaking waves. Noblesse et al.[20, 21] and Delhommeau et al. [10] have studied the behaviour of the breaking waves depending on the bow geometry and the Froude number. Similary, numerical simulations of two-phases flow are still being developed. Ma et al. [18], Carrica et al. [3], Castro et al. [4] describe a sub-mesh model coupled with a two phases RANS model to resolve the flow and obtain a quantitative numerical prediction of the distribution of void fraction around the ship hull. However, these simulations relied upon simple entrainment models or arbitrarily set bubble sources. Moraga et al. [19] developed a model for locating regions of high void fraction using bubble distributions observed by Deane and Stockes [5] during breaking waves. 


\section{Experimental Set-up}

\subsection{The wave and current flume tank}

Experiments have been carried out at the Ifremer (French Research Institute for Exploration and Exploitation of the Sea) wave and current flume tank (figure 1). The tank is $\mathrm{U}=0.1$ to $2.2 \mathrm{~m} / \mathrm{s}$. The flow turbulence in the tank is $3 \%$ by the use of flow straighteners. A wave generator (figure 1 right), composed of eight independent displacement paddles, each $0.5 \mathrm{~m}$ wide and $500 \mathrm{~mm}$ deep, can be easily moved between an upstream or a downstream surface position to create waves propagating with or against the current. When the wave generator is used to generate waves with the current, it increases the
turbulence level to $15 \%$ close to the free surface. The system is able to generate regular
and irregular waves with a frequency range between 0.5 and $2 \mathrm{~Hz}$ and a maximum ampli-
tude of $280 \mathrm{~mm}$ with a current speed up to $0.8 \mathrm{~m} / \mathrm{s}$. Measurements have revealed that the
resulting reflection coefficient was less than $12 \%$ for all the usual periods and amplitudes.
A side observation window of $8 * 2 \mathrm{~m}^{2}$ placed on one side of the tank allows users to When the wave generator is used to generate waves with the current, it increases the
turbulence level to $15 \%$ close to the free surface. The system is able to generate regular
and irregular waves with a frequency range between 0.5 and $2 \mathrm{~Hz}$ and a maximum ampli-
tude of $280 \mathrm{~mm}$ with a current speed up to $0.8 \mathrm{~m} / \mathrm{s}$. Measurements have revealed that the
resulting reflection coefficient was less than $12 \%$ for all the usual periods and amplitudes.
A side observation window of $8 * 2 \mathrm{~m}^{2}$ placed on one side of the tank allows users to When the wave generator is used to generate waves with the current, it increases the
turbulence level to $15 \%$ close to the free surface. The system is able to generate regular
and irregular waves with a frequency range between 0.5 and $2 \mathrm{~Hz}$ and a maximum ampli-
tude of $280 \mathrm{~mm}$ with a current speed up to $0.8 \mathrm{~m} / \mathrm{s}$. Measurements have revealed that the
resulting reflection coefficient was less than $12 \%$ for all the usual periods and amplitudes.
A side observation window of $8 * 2 \mathrm{~m}^{2}$ placed on one side of the tank allows users to When the wave generator is used to generate waves with the current, it increases the
turbulence level to $15 \%$ close to the free surface. The system is able to generate regular
and irregular waves with a frequency range between 0.5 and $2 \mathrm{~Hz}$ and a maximum ampli-
tude of $280 \mathrm{~mm}$ with a current speed up to $0.8 \mathrm{~m} / \mathrm{s}$. Measurements have revealed that the
resulting reflection coefficient was less than $12 \%$ for all the usual periods and amplitudes.
A side observation window of $8 * 2 \mathrm{~m}^{2}$ placed on one side of the tank allows users to bow geometry, one can obtain the characteristics of the wave generated and estimate the properties of the plunging jet causing the air entrainment. Knowledge of these properties then enables us to calculate the quantity of air entrained, the size of the bubbles generated as well as the penetration in depth. However, the air entrainment by the bow wave described above is valid only in calm water. These models are thus more relevant to study the air-water exchange close to the free surface than the bubble sweep-down phenomenon occuring deeper under more severe conditions. On the other hand, the body-wave interaction in the bow vicinity of the ship is the origin of bubble clouds [9]. Therefore, it is overriding to take into account the hull geometry characteristics as well as the sea state

In this work, the bubble sweep-down phenomenon around three ship models with different bow geometries is studied within the same experimental protocol [9] in order to reproduce the ships behaviour in terms of generation and propagation of air bubbles. The first part of this paper presents the experimental set-up allowing the reproduction of the the flow on a plane around the bow is then described. The second part is devoted to the characterization of bubble cloud dynamics for the three models in term of area, maximal depth and vertical velocity for different configurations. The final part focuses on PIV and POD (Proper Orthogonal Decomposition) data analysis in order to identify the flow behaviour during air entrainment.

These models provide a lot of information: from the forward speed of a ship and the 
observe the behavior of the ship model during trials. Unlike towing tanks where models are free to heave and pitch, the model has been fixed on a motion generator (hexapod) enabling the reproduction of any motion in the 6 degrees of freedom. The free surface elevation is measured by a wave gauge system placed one meter upstream from the ship bow.
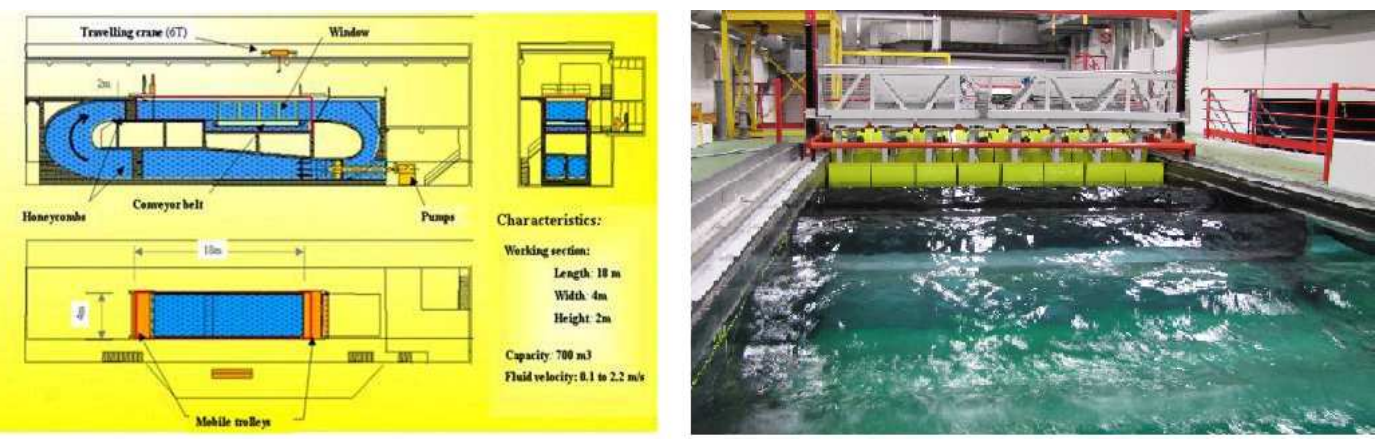

Figure 1: Shematic view of the wave and current circulating tank (left) and a view of the wave generator with regular waves (right).

\subsection{Ship Models and Similarity Issues}

Tests have been conducted on three ship models at a scale of $1 / 30$ to study the effect of the bow geometry on the bubble sweep-down phenomenon. The first model is a classic bow of the French oceanographic research ship Pourquoi pas? (PP). The second is an Inverted Bow (IB) design. It has been designed so as to maintain the overall length, beam and draft of the PP. The third model (Thin Bow, TB) has been designed as a fishing boat with the same overall length and beam but with a higher draft. For the three models, the length between perpendiculars is $3.13 \mathrm{~m}$ with a beam of $0.67 \mathrm{~m}$. The classic bow of the PP and the IB have a draft of $0.182 \mathrm{~m}$ and a block coefficient $C_{B}$ of 0.577 , whereas the draft of the TB is $0.24 \mathrm{~m}$ with a block coefficient of 0.442 . The characteristics of the different hull geometries at model and full scales are resumed in the table 1. Figure 2 shows the photos and the CAD images of the three models from different angles of vue. Trials have been carried out on the front part only ( $1 / 3$ of the total length of the model) to avoid disturbances coming from the model stern (figure 4 left).

\begin{tabular}{|c|c|c|c|c|c|c|}
\hline & \multicolumn{2}{|c|}{ Pourquoi pas? } & \multicolumn{2}{c|}{ Inverted Bow } & \multicolumn{2}{c|}{ Thin Bow } \\
\hline & model scale & full scale & model scale & full scale & model scale & full scale \\
\hline $\operatorname{Lpp}(m)$ & 3.13 & 94 & 3.13 & 94 & 3.13 & 94 \\
\hline Beam $(\mathrm{m})$ & 0.67 & 20 & 0.67 & 20 & 0.67 & 20 \\
\hline Draft $(\mathrm{m})$ & 0.182 & 5.46 & 0.182 & 5.46 & 0.24 & 7.2 \\
\hline$C_{B}$ & 0.577 & 0.577 & 0.577 & 0.577 & 0.442 & 0.442 \\
\hline
\end{tabular}

Table 1: The characteristics of the different hull geometries at model and full scales. 

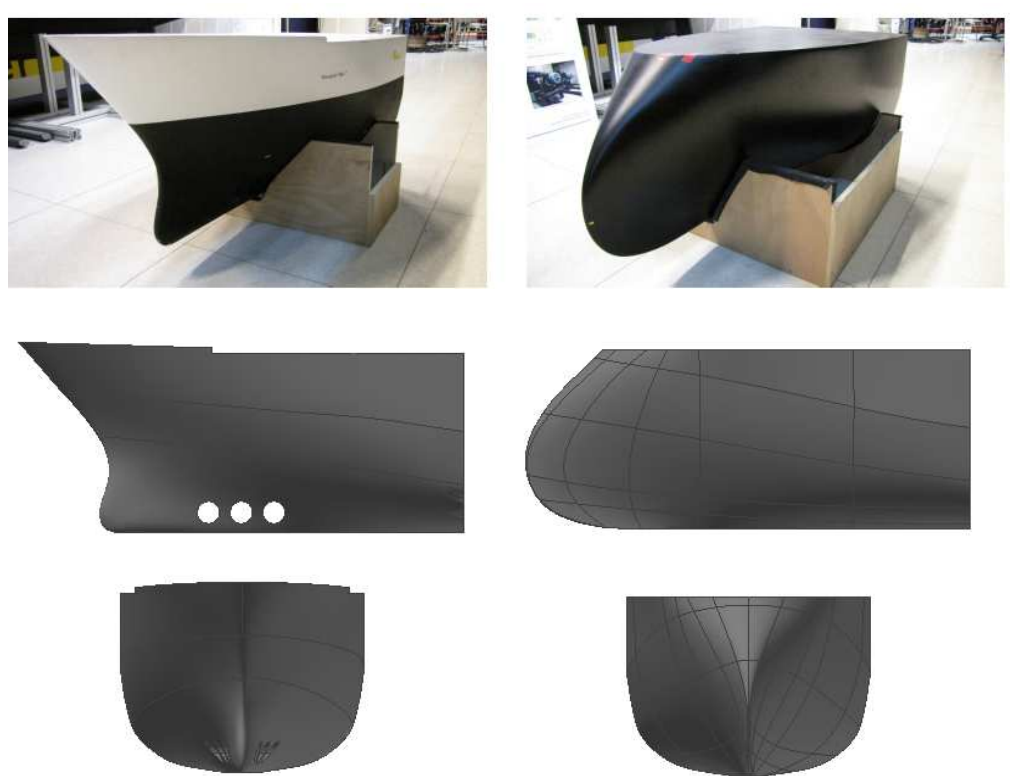
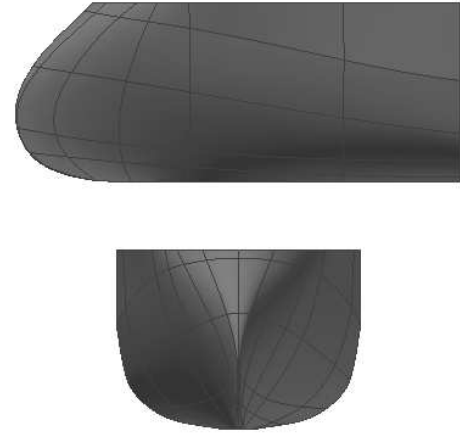
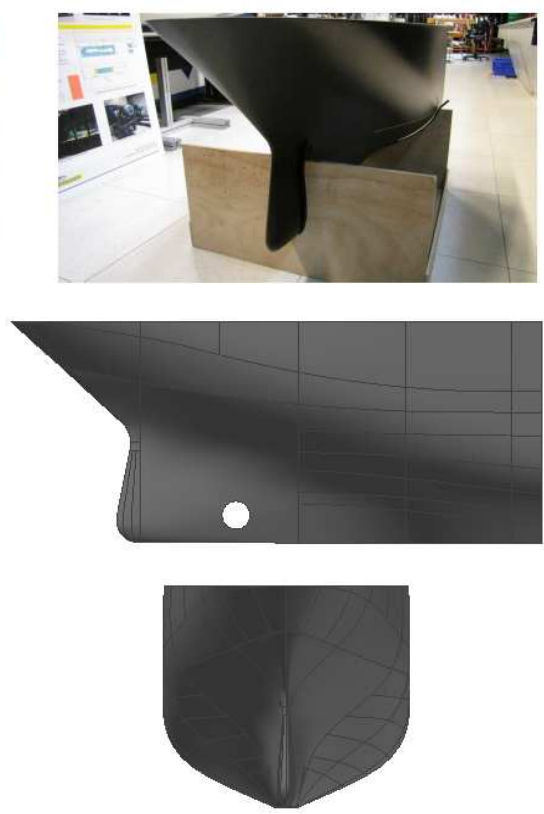

Figure 2: Top to bottom, from left to right: photos and CAD images from a side view and a front view of the classic bow of the PP, the IB and the TB. reproducing navigation conditions and sea states during which the phenomenon has been observed on the French oceanographic research vessel PP, during the acoustic survey Essbulles in 2005 [6]. The main conditions during this survey are: a ship speed of 8knots, a wave with a wavelength of $109 \mathrm{~m}$, a significant wave height $\mathrm{Hs}$ of $2.8 \mathrm{~m}$, and a wave period Tp of 8.4s.

Regarding the generation and propagation of air bubbles, the parameters involved are related to the propagation of waves and ship motions. Thus, the experimental study demands that the Froude similitude be respected for a good representation of the free surface influence. In this case, the Froude number, $F r=U^{2} / g . L p p=0.203$, where U is the ship speed, g the gravity and Lpp the model length. However, it is impossible to comply both Froude and Reynolds similarities preserving the physical characteristics of the fluid. The Reynolds number ( $R e=U L_{p p} / \nu$; where $\nu$ is the kinematic viscosity) is then largely lower during tank trials comparing to the flow around a full scale vessel by a factor of $100\left(R e_{\text {model }}=2.1 \cdot 10^{6} ; R e_{\text {real }}=3.4 \cdot 10^{8}\right)$. The induced viscous effects mainly affect the boundary layer very close to the hull and can delay the onset of turbulent flow which is compensated in the flume tank by a strong turbulent intensity. On the other hand, the Froude similarity is also problematic for the generation and propagation of bubbles in the flow. Bubble dynamics are related to ensure the stability of the inertial forces but also to the surface tension (and therefore the Webber number $W e=\rho L U^{2} / \sigma$, where $\rho$ is the fluid density, $\mathrm{L}$ is the characteristic length and $\sigma$ is the surface tension). The surface tension is relatively more or less significant depending on the characteristics 
of the wave itself controlled by the dimension of the model. As with the Reynolds number, the Webber number can not be preserved $\left(W e_{\text {model }}=2.5 \cdot 10^{3} ; W e_{\text {real }}=2.3 \cdot 10^{5}\right)$. We have in mind that by using ship Froude scaling, bubble Froude, Reynolds and Weber scaling is inevitably violated. Then the following bubble dynamics analysis performed in the flume tank may be not exactly the same as at real scale. However, the three bow geometries are tested under the same flow configurations and the following results are expected to provide to some significant informations associated with flow and body interaction leading to the bubble sweep-down phenomenon. Finally, the Froude similarity imposes a wave amplitude and flow velocity lower than at real scale. Therefore the shear forces in the flow close to the model and the amount of air entrainment beneath the surface will be significantly reduced. To limit the scale effects as much as possible, the model chosen is as large as possible based on the flume tank characteristics (lateral space, wave and current generation). Hence for the 1/30 scale ship model, the flow velocity in the flume tank is fixed at $0.75 \mathrm{~m} / \mathrm{s}$, the wave amplitude at $33 \mathrm{~mm}$ with a frequency of $0.85 \mathrm{~Hz}$. A synthesis of all the main experimental parameters is given in table 2 .

\begin{tabular}{lllllllll}
\hline & $L_{p p}(m)$ & $U(m / s)$ & $F r$ & $R e$ & $W e$ & $H s(m)$ & $T p(s)$ & $\lambda(m)$ \\
\hline Ship & 94 & 4.12 & 0.203 & $3.4 \cdot 10^{8}$ & $2.5 \cdot 10^{5}$ & 2.8 & 8.4 & 109 \\
Model & 3.13 & 0.75 & 0.203 & $2.1 \cdot 10^{6}$ & $2.3 \cdot 10^{3}$ & 0.066 & 8.4 & 3.63 \\
\hline
\end{tabular}

Table 2: Summary of the main parameters at both real and model scales.

In this study, it was assumed that the three ships have the same behaviour in terms of motions (heave and pitch) at sea. The imposed motions are determined by means of a numerical model using the computer program FREDYN. A nonlinear strip theory approach is used to compute the hydrodynamic forces acting on the hull. In this head sea configuration, the only significant motions are heave and pitch. Surge motions are here considered insignificant ( $\leq 0.1 \mathrm{~m}$ at full scale). After converting results to the present $1 / 30$ scale ship model by Froude scaling, we finally obtain $20 \mathrm{~mm}$ for heave and 2 for pitch motions. A key point in the experiments is the synchronization of the waves and motions [9]. To achieve this, hexapod motions have been synchronized with the wave and the synchronism has been verified a posteriori (see Figure 3). 


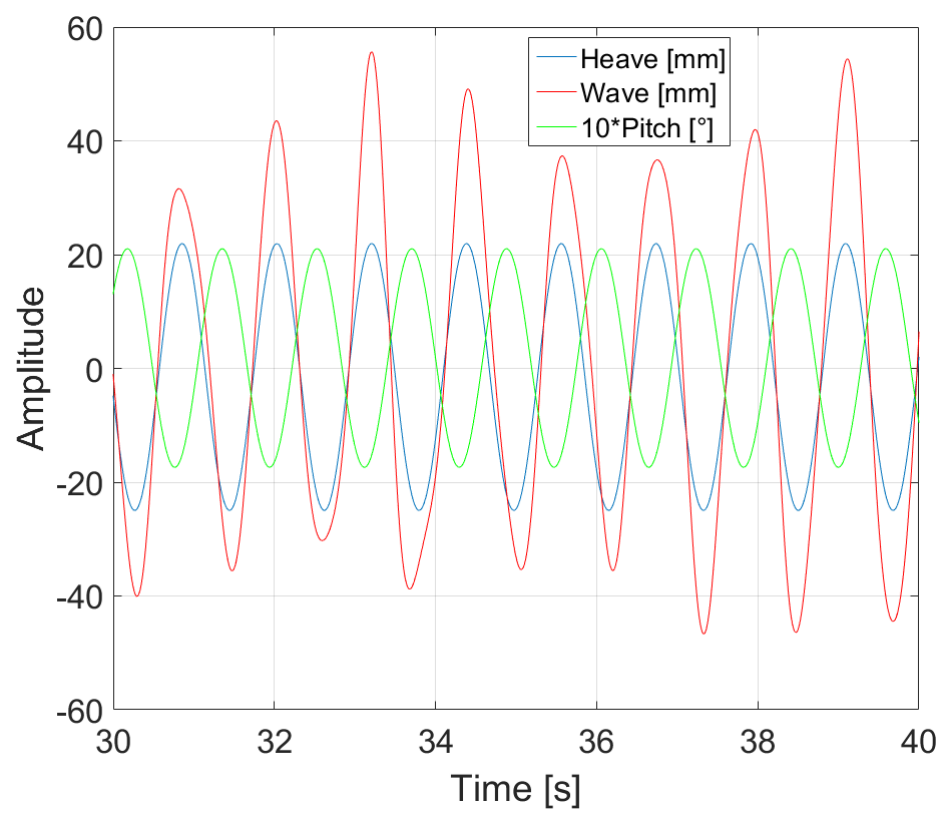

Figure 3: Exemple of imposed ship motions and wave signals on the 1/30 bow ship model.

\subsection{Flow measurements}

To characterize the flow around the bow, measurements have been taken by means of a 2D PIV system on a vertical plane along the $\mathrm{x}$ axis and located at $\mathrm{y}=0 \mathrm{~mm}$ (Figure 5 ). For these measurements, the tank has been seeded by silver-coated glass particles of $10 \mu \mathrm{m}$ diameter. Illumination has been provided by a standard, frequency-doubled, double-cavity Nd:YAG laser with a pulse energy of up to $120 \mathrm{~mJ}$ per pulse using an excitation wavelength of $532 \mathrm{~nm}$ (Figure 4 right). The laser sheet is emitted in the water, by the use of an optical system mainly composed of cylindrical lenses mounted on a vertical laser guiding arm in order to generate a light sheet on vertical plane with a low interval time $\mathrm{t}=1200 \mu \mathrm{s}$, which also corresponds to the time between the double frame PIV images. The camera (Hi-sense CCD camera of $1600 * 1200$ pixels ${ }^{2}$ ) recording PIV images has a 8.5fps to obtain 10 velocity maps per wave period. The distance between the camera and the laser sheet is $2.2 \mathrm{~m}$; the camera is located perpendicularly to the laser sheet. DynamicStudio software from Dantec Dynamics has been used for PIV image processing. The instantaneous velocity vector fields have been obtained using an Adaptive PIV algorithm with an interrogation window size of $32 * 32$ pixels $^{2}$ and an adjacent windows of $25 \%$ overlapped. Streamwise (x-direction) and transverse (ydirection) velocity components are then available on a $2 \mathrm{D}$ regular mesh grid of $\left(n_{x} \mathrm{x} n_{z}\right)$

$155=(66 \mathrm{x} 49)$ points with a similar grid of $8 \mathrm{~mm}$ in each direction. The physical dimensions of the PIV plane are $528 * 396 \mathrm{~mm}^{2}$. The experimental set-up is summarized in Figure 6 . 

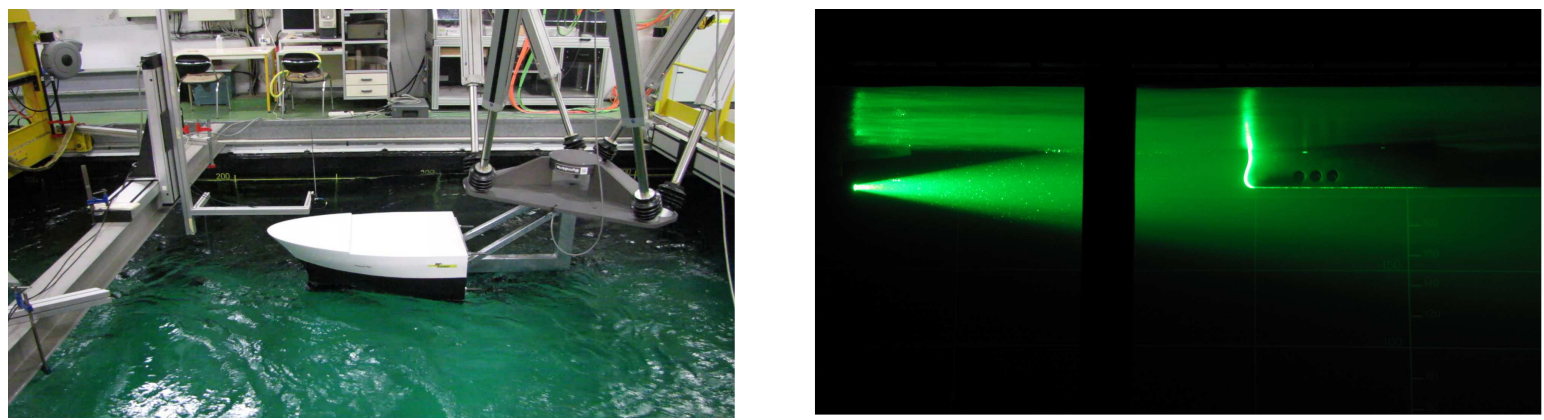

Figure 4: View of the experimental setup with the PP bow model (left) and side view of the laser sheet emitted in front of the bow (right).
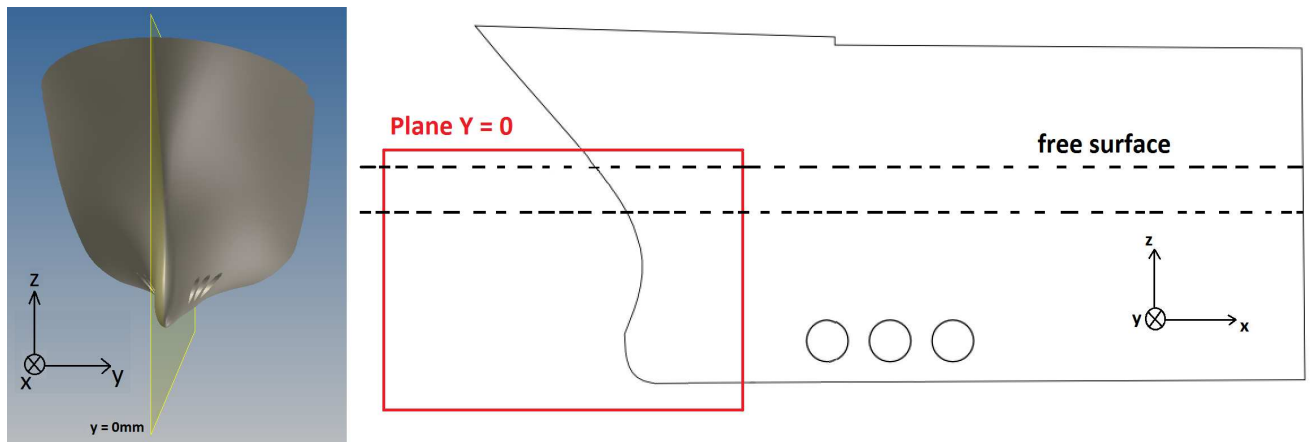

Figure 5: Presentation of the PIV plane location (front and side view).
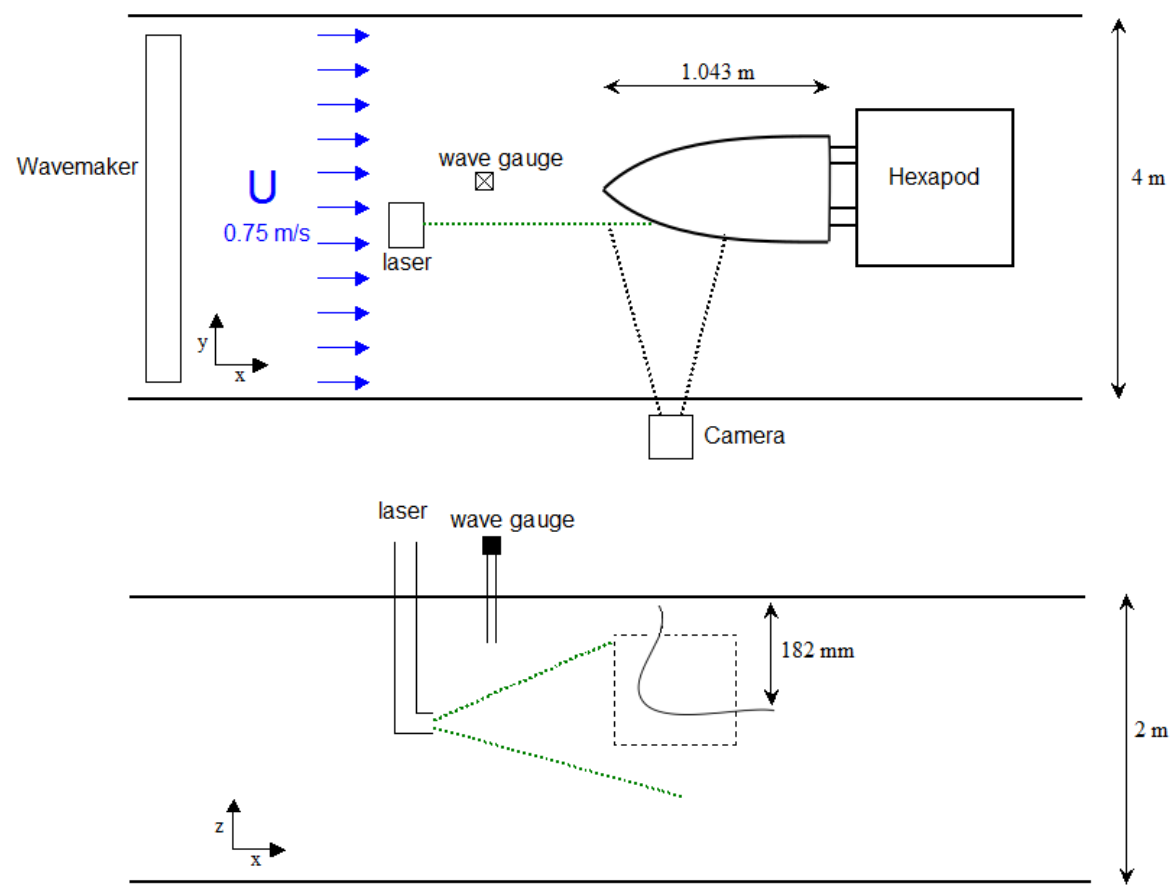

Figure 6: Schematic view of the experimental set-up in the wave and current circulating tank, top and side view showing the PIV system location. 


\subsection{Tested Configurations}

To highlight the physical phenomena that lead to bubble sweep-down, four configurations have been tested to apprehend the influence of each parameter (current, wave motion and model ship motion) on these phenomena. Table 3 gives the characteristics of each tested configuration which can be submitted to either current (flow velocity of $U=0.75 \mathrm{~m} / \mathrm{s})$, and $/$ or regular waves $\left(33 \mathrm{~mm}\right.$ amplitude and $f_{0}=0.85 \mathrm{~Hz}$ frequency) and/or model ship motion ( $2^{\circ}$ pitch and $20 \mathrm{~mm}$ heave at $\left.0.85 \mathrm{~Hz}\right)$, forced by the hexapod. Note that for the fourth configuration, the model has been submitted to the current, as well as synchronized waves and motions.

\begin{tabular}{|c|c|c|c|}
\hline Configuration & $\begin{array}{c}\text { Current } \\
(U=0.75 \mathrm{~m} / \mathrm{s})\end{array}$ & $\begin{array}{c}\text { Regular waves } \\
(\mathrm{H}=33 \mathrm{~mm} ; \mathrm{f}=0.85 \mathrm{~Hz})\end{array}$ & $\begin{array}{c}\text { Model ship motion } \\
\left(20 \mathrm{~mm}, 2^{\circ}, \mathrm{f}=0.85 \mathrm{~Hz}\right)\end{array}$ \\
\hline 1 & $\mathrm{x}$ & - & - \\
\hline 2 & $\mathrm{x}$ & $\mathrm{x}$ & - \\
\hline 3 & $\mathrm{x}$ & - & $\mathrm{x}$ \\
\hline 4 & $\mathrm{x}$ & $\mathrm{x}$ & $\mathrm{x}$ \\
\hline
\end{tabular}

Table 3: Detail of characteristics the four tested configurations.

In the following, the four configurations will be investigated to study the bubble sweepdown phenomenon around the three ship models in terms of bubble cloud occurrence frequency and of bubble cloud dynamics. For the study of the flow around the bow ships, we will focus only on the configuration 2 and configuration 4 . \\ 3 Bubble clouds characterization}

Through these experimental configurations, two different air entrainment mechanisms have been detected [9]. The first one is the air entrainment by vortex shedding due to the interaction between the incoming turbulent flow and the bow wave which generates a small air cavity until the black entrapment (see figure 7). The second type of air entrainment is due to breaking waves resulting from interaction between the incoming wave and a reflected one on the bow and/or the impact between the bow and the free surface (see figure 8 and 9). The two mechanisms have been observed and identified for the three ship models. In the following, we compare the occurrence frequency of the two mechanisms around the three models. 

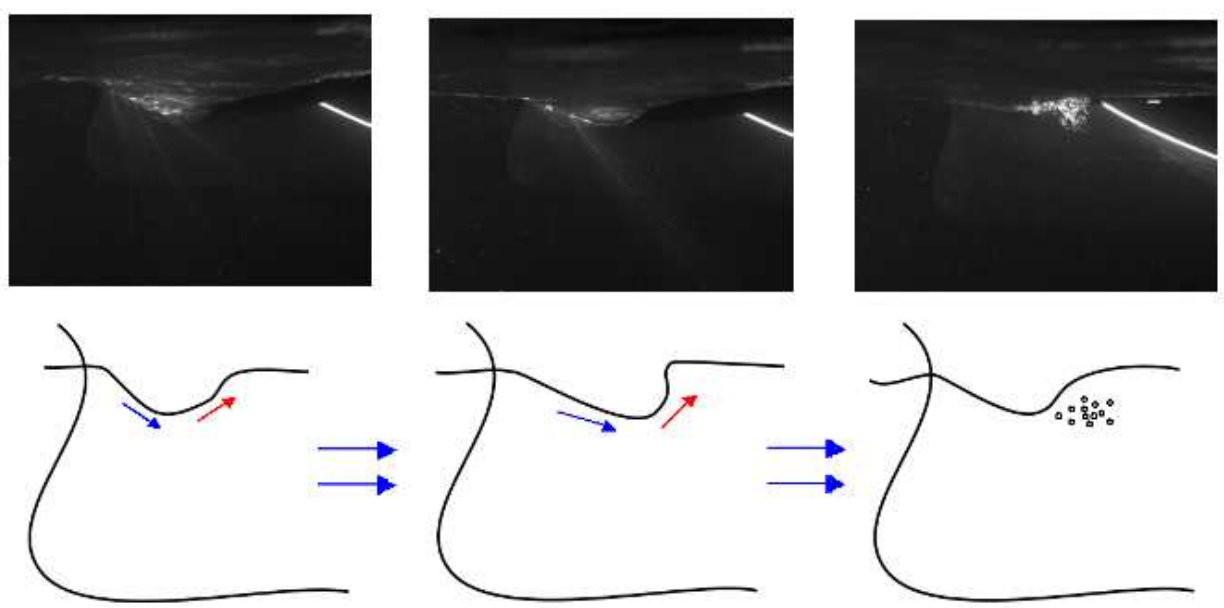

Figure 7: Description of the air entrainment mechanism by vortex shedding cloud, from the free surface distortion to the air bubble generation. Top: raw images. Bottom: schematic representation of the mechanism.
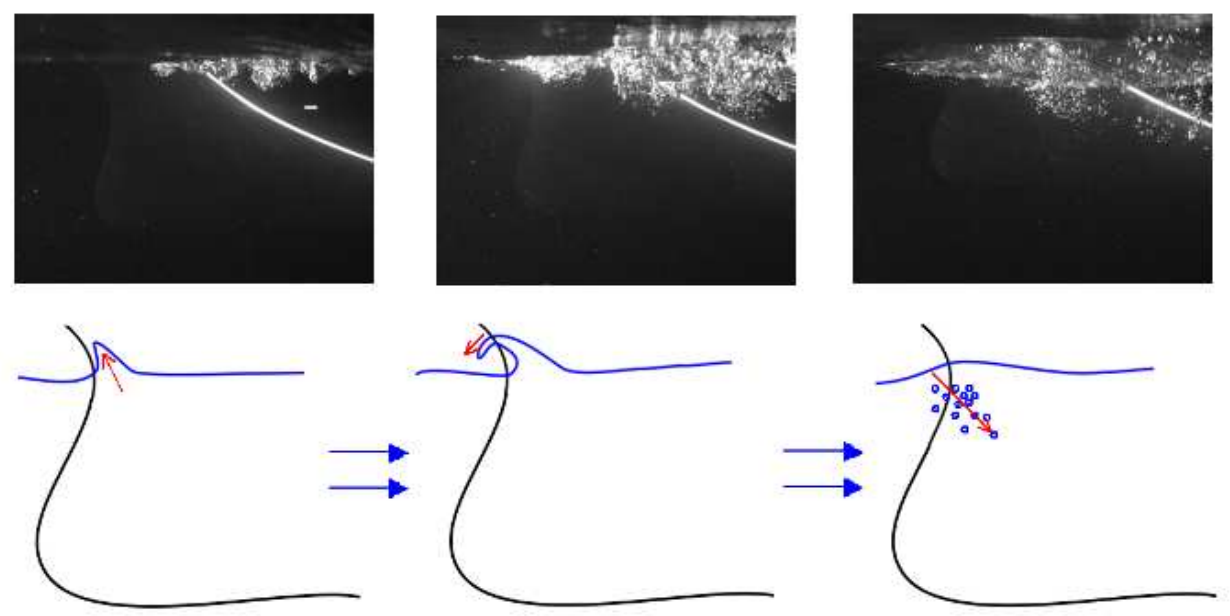

Figure 8: Description of the air entrainment mechanism by breaking wave. Top: raw images. Bottom: schematic representation of the mechanism.
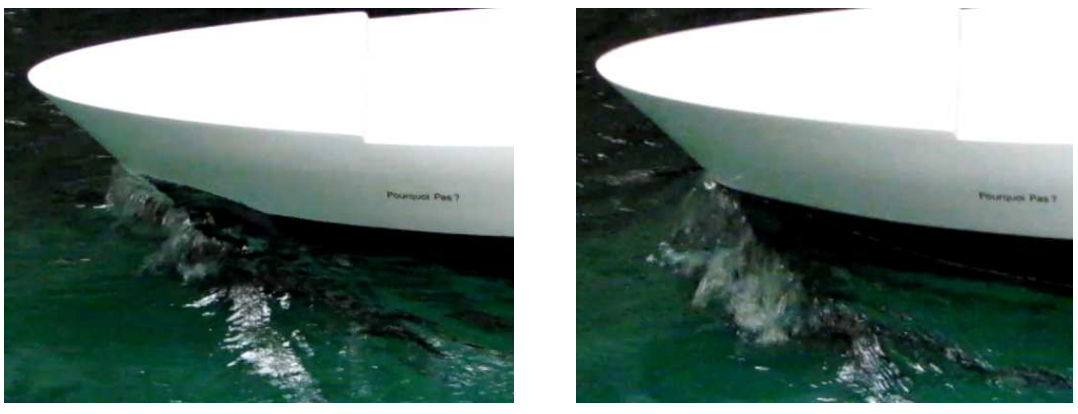

Figure 9: Visualization of the breaking wave at the bow vicinity of the PP 


\section{$3.1 \quad$ Occurrence frequency of bubble clouds}

Due to the difficulty in identifying such a phenomenon nevertheless characterized by a dominant frequency, the occurrence frequency of the two air entrainment mechanisms around the three models has been first computed by a manual count for each of the four basic configurations. This corresponds to the number of events (vortex shedding or breaking wave) divided by the acquisition time of 3 minutes. Images have been taken by an underwater camera with a frequency of $10 \mathrm{~Hz}$. This method allows to observe and identify each air entrainment mechanism for the different configurations. The number of bubble events have been counted every 30 seconds over the whole available time duration (180s), which enabled us to determine an 'equivalent' frequency corresponding to the passage of bubble cloud events and to check the convergence of the results. The results are shown in figure 10 where $\mathrm{f}$ is the occurrence frequency of bubble clouds and $f_{0}$ is the inlet frequency of wave and ship motions. For the configuration 2, 3 and 4, the dimensionless frequency of bubble clouds $\left(\mathrm{f} / f_{0}\right)$ is presented. As the configuration 1 is only with current, we present its result in term of $\mathrm{f}$ only.

The following has been observed.

- The occurence frequency of bubble generation by vortex shedding cloud remains constant for the four configurations and for every type of bow. For the PP ship model, this frequency is double that of the inverted and thin bows. It is about $0.2 \mathrm{~Hz}$ for the classic bow of PP and about $0.08 \mathrm{~Hz}$ for the inverted and thin bows. It is due to the flairing of the bow of the PP that allow to generate more small air cavities and consequently more air bubble entrapment;

- The inverted and thin bows have almost the same behaviour for the four configurations with slightly more breaking wave clouds around the TB for the configurations 2 and $3 ;$

- The bubble clouds occurence frequency decreases strongly for both thin and inverted bows compared to the classic bow of PP in the configurations 2 (Current + waves) and 4 (Current + waves + motions). The occurrence frequency of bubble clouds (by vortex shedding and breaking waves) obtained for the configuration 2 are $0.69 f_{0}$ for the classic bow, against $0.3 f_{0}$ and $0.24 f_{0}$ for the thin and IBs repectively;

- In configuration 3, we can see that the occurrence frequency of bubble generation by vortex shedding cloud is equal to the occurrence frequeny of bubble clouds by breaking waves for the classic bow of the PP. However, we have more bubble clouds by breaking waves than bubble clouds by vortex shedding for the inverted and thin bows. 

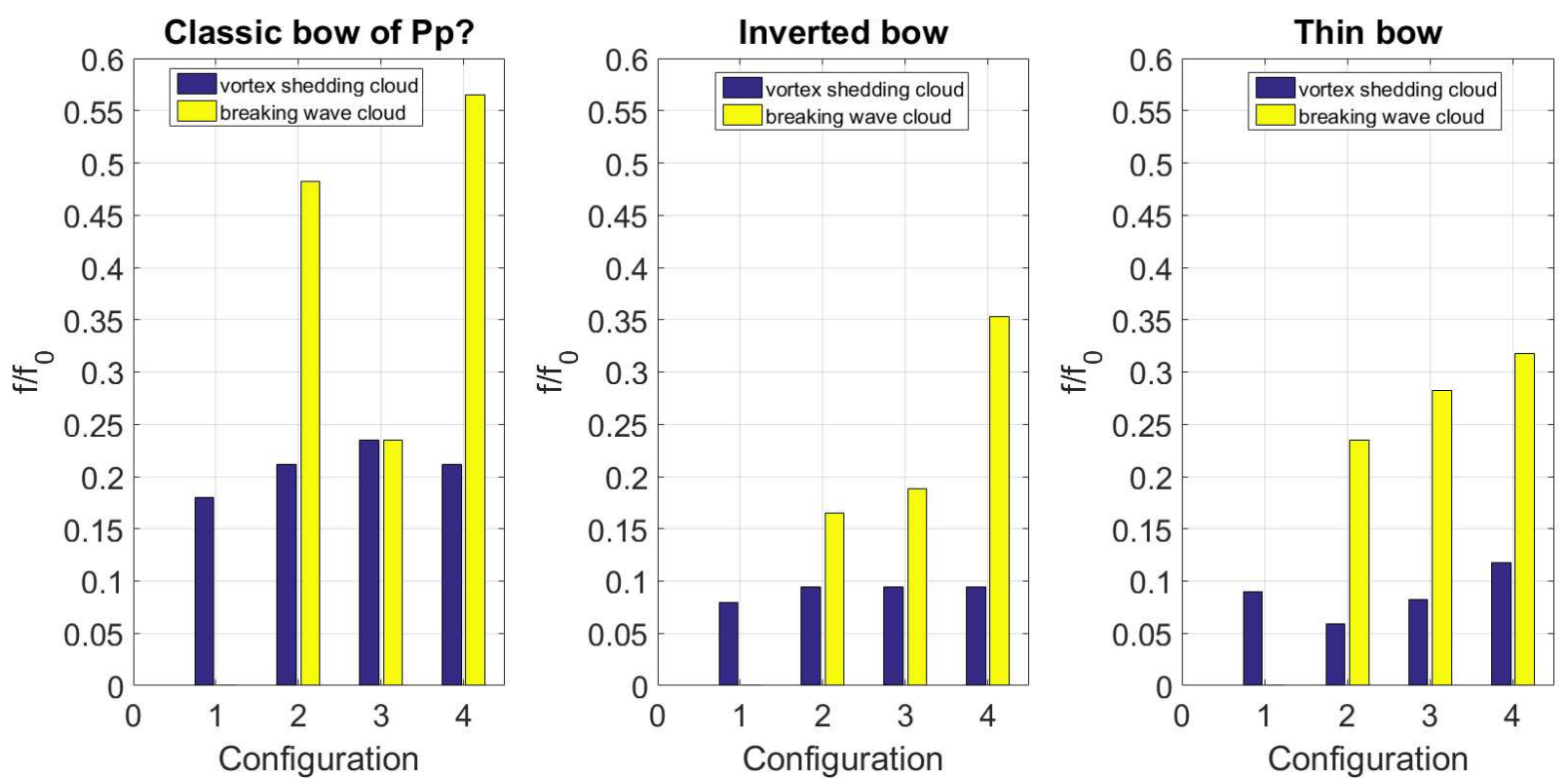

Figure 10: Occurrence frequency of bubble clouds for all the configurations around the three ship bow models.

From all these observations, we can note that the wave is the main parameter acting on bubble cloud generation around the classic bow of the PP. For the IB, only the combination between waves and ship motions allows to generate a significant number of bubble clouds. Waves and ship motions play the same role for the TB. We have nearly the same occurrence frequency of bubble clouds in the configuration 2, 3 and 4 . All these results show that the flairing of the classic bow of the PP mainly acts on bubble generation.

\subsection{Dynamic properties of bubble clouds}

The main goal of this study is to characterize the bubble sweep-down phenomenon around the ship models in terms of bubble cloud dynamics to better understand the influence of the bow geometry on the phenomenon. In order to compare the bubble clouds properties in terms of area, maximum depth and vertical velocity for the three models, an image processing method via a gray scale analysis has been developed to detect and follow the bubble clouds [8]. The bubble clouds occur in 3D, so we only measure a portion of the cloud that is not easily quantified due to the difficulties coming from the depth of field. However, the aim of this study is to reproduce the trials in the same conditions and in the same measure plane around the three ship models in order to compare the behaviour of the bubble clouds around them. For that purpose, each image is treated via a gray level analysis: First, the raw image is converted to a binary image taking into account a first gray level. This binary image allows us to detect the free surface and the bow model in the front due to the reflection of the laser sheet. The 
region of the biggest bubble cloud is then detected. A second loop with a second gray level threshold is applied in order to obtain a maximum of information in the bubble cloud area. Different thresholds have been tested in order to detect the bubble cloud accurately and it was shown that the threshold variations have very little influence on the results presented in the following.

This method allows to better characterize and quantify the two air entrainment mechanisms and the dynamic of the bubble clouds. An example of the detection and evolution of a bubble cloud generated by breaking waves is shown in figure 11. Figure 12 shows the main bubble cloud properties for a selected image: the area $\left(A_{\text {cloud }}\right)$ in white, the maximal depth $\left(Z_{\max }\right)$ and the vertical velocity $\left(W_{\text {cloud }}\right)$ calculated from the centroid of the identified region (determined from the bubble cloud center locations). In some cases, where the entire bubble cloud is not completely present in the image (especially at the end of the wave period), the bubble cloud center position is badly detected (see figure 13). Therefore, the vertical velocity calculation is not fully validated but sufficient to compare the behaviour of the three ship models in term of performance against the bubble generation.

1
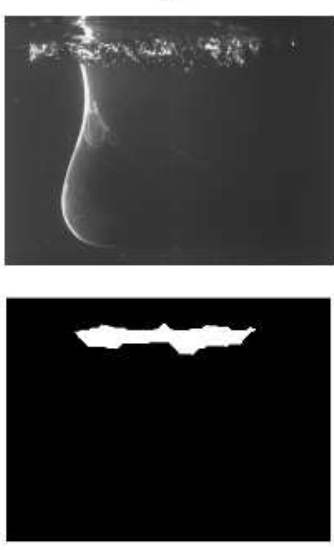

5
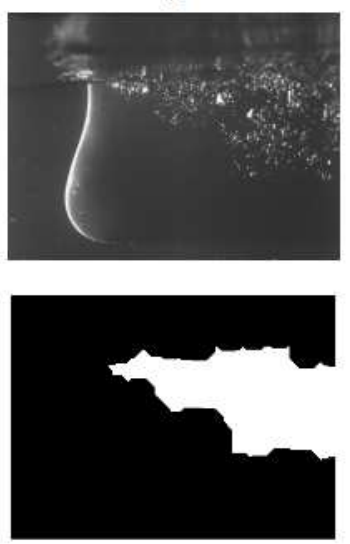

2
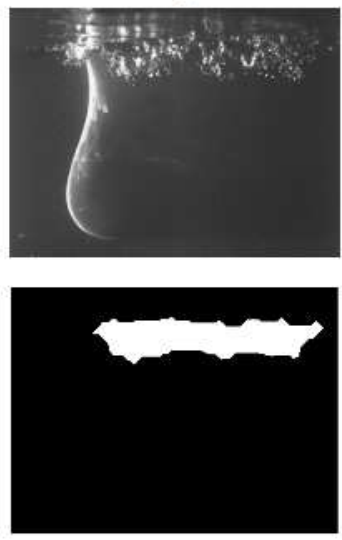

6
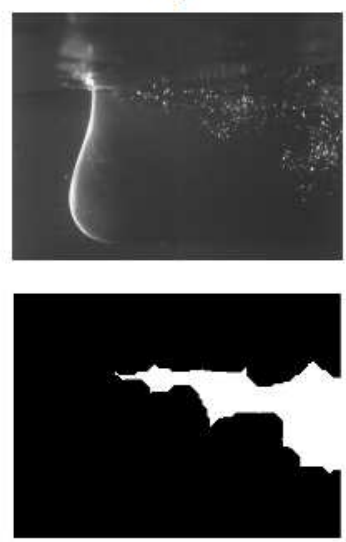

3
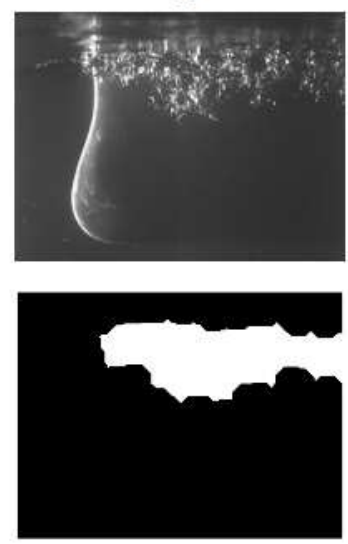

7
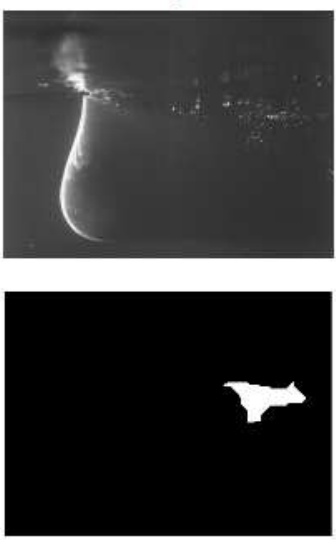

4
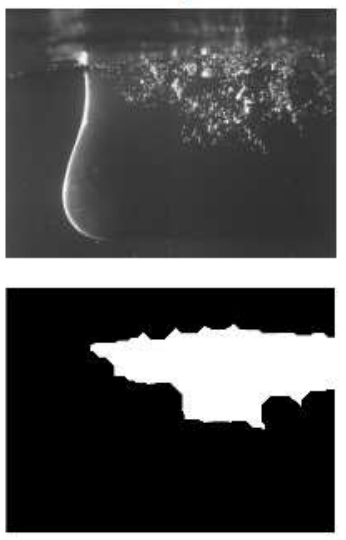

8
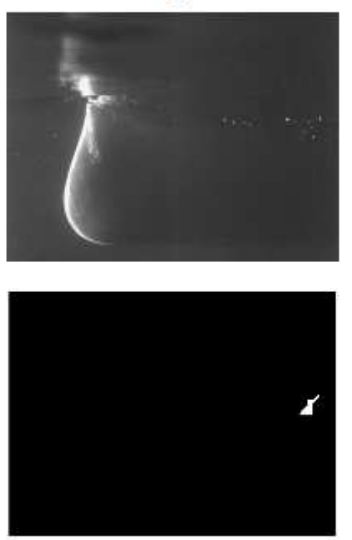

Figure 11: Detection and evolution of a bubble cloud during a wave period in the configuration 2 around the classic bow of PP. 


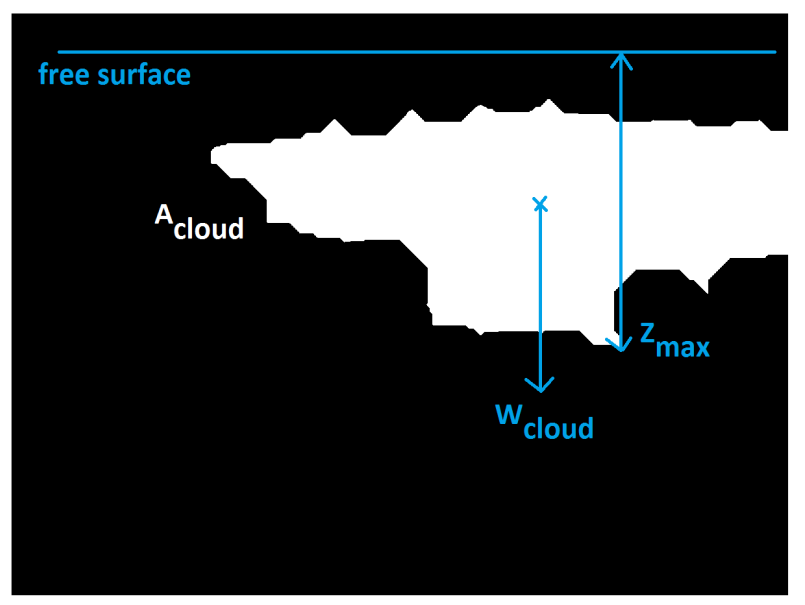

Figure 12: Dynamic properties of a bubble cloud: the area $\left(A_{\text {cloud }}\right)$ in white, the maximal $\operatorname{depth}\left(Z_{\max }\right)$ and the vertical velocity $\left(W_{\text {cloud }}\right)$.

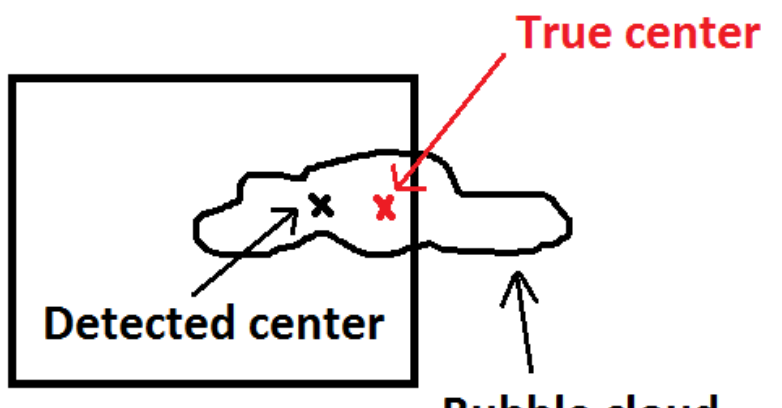

\section{Bubble cloud}

Figure 13: schematic view of a case where a bubble cloud is not entirely present in the PIV image represented as a black square.

Figure 14 to Figure 17 show the dimensionless bubble properties around the three models and for all basic configurations. The adimensionalization has been done with:

- $A_{0}$ is the area defined by the draft and the length $l=0.2 L_{p p}=0.626 \mathrm{~m}$. It is about $0.152 m^{2}$ for the TB and $0.113 m^{2}$ for PP and IB,

- $Z_{0}$ is the draft of the ship model. It is about $0.243 \mathrm{~m}$ for the TB and $0.182 \mathrm{~m}$ for the $\mathrm{PP}$ and IB,

- $\mathrm{U}=0.75 \mathrm{~m} / \mathrm{s}$ is the current velocity.

The abscissa of each figure corresponds to the dimensionless time $\mathrm{t} / \mathrm{T}$, where $\mathrm{T}$ is the wave and motions period $(\mathrm{T}=1 / 0.85=1.18 \mathrm{~s})$. Each line in these figures shows the evolution of the mean value of all significant bubble clouds detected for each type of bow, while a dotted line corresponds to the maximal property evolution of the same 'extreme' bubble cloud to have an idea of how far a bubble cloud can reach in terms of maximal depth, maximal area and maximal vertical velocity. A significant bubble cloud is defined 


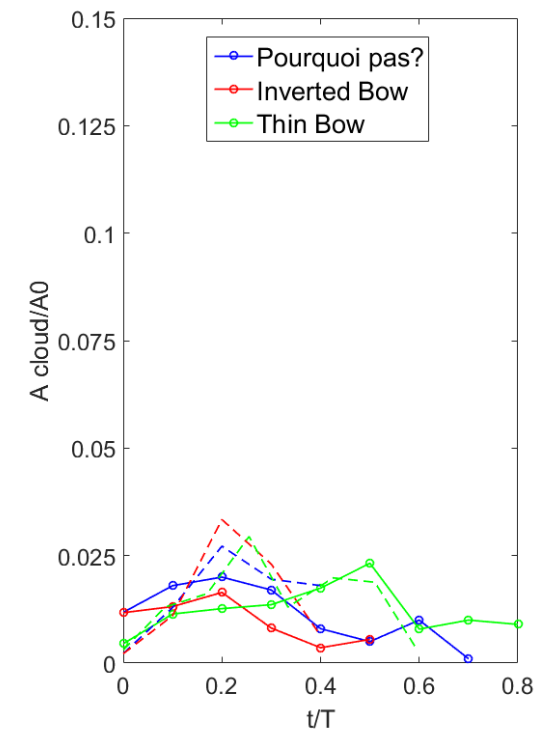

- For the configuration 1 with current only (Figure 14), the instant $\mathrm{t}=0$ corresponds to the first aeration detection for every cloud. During 3 minutes of acquisition, we detect 11 bubble clouds around the PP, 10 around the IB and 10 around the TB. The bubble clouds mean area reaches a value of $0.025 A_{0}$ for the TB and $0.02 A_{0}$ for the classic and inverted bows. The maximal mean depth is about $0.18 Z_{0}$ for the IB and slightly greater than $0.2 Z_{0}$ for the classic and thin bows. The vertical velocity is close to zero in the first half of the life of the clouds and then tends towards a positive value, the bubble clouds rising to the surface. Bubbles clouds have not some important area and vertical velocities in this configuration compared to other ones. So there is no potential influence on the acoustic measurements in this case.

Figure 14: The bubble clouds properties (area, maximal depth and vertical velocity) in configuration 1 with current only around the three ship models. Each line corresponds to the mean evolution of bubble clouds properties, the dotted line corresponds to the maximal evolution of an 'extreme' bubble cloud property.

- For the configuration 2 with current and waves (Figure 15), the instant $\mathrm{t}=0$ corresponds to the peak of the incoming wave at the bow. During the 3 minutes of acquisition, we detect 20 bubble clouds around the PP, 7 around the IB and 12 around the TB. The mean cloud area reaches a value of $0.05 A_{0}$ for the classic bow of PP, $0.013 A_{0}$ for the IB and $0.016 A_{0}$ for the TB. This decrease in area is due to the fact that breaking waves have small velocities around the thin and inverted bows which generate fewer significant clouds in terms of area. The mean depth 
reaches a value of $0.48 Z_{0}$ for the classic bow of the $\mathrm{PP}$, it is slightly greater than $0.25 Z_{0}$ for the IB and slightly less than $0.28 Z_{0}$ for the TB. This decrease is also due to the smaller clouds around the two last bows. In other words, the inverted and thin bows generate waves with less impact jet velocity compared to the clas-

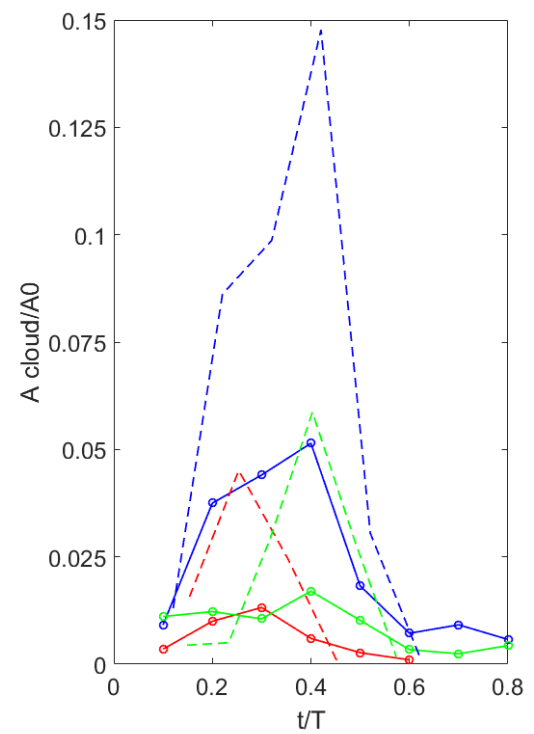

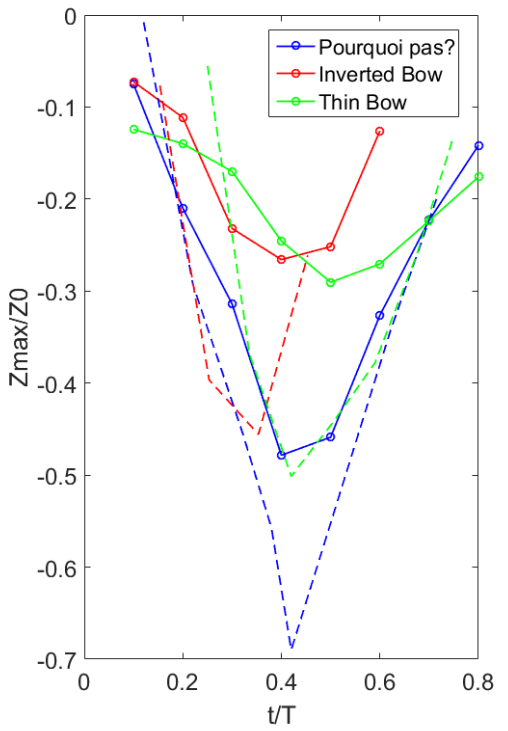

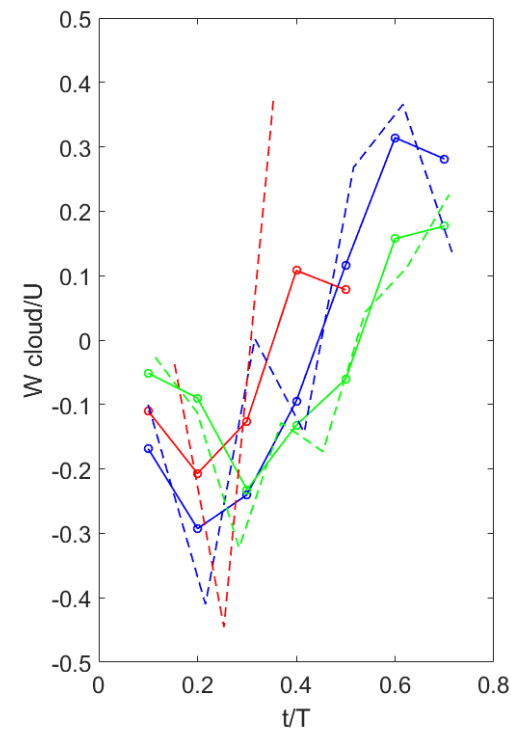

Figure 15: The bubble clouds properties (area, maximal depth and vertical velocity) in configuration 2 (current and waves) around the three ship models. Each line corresponds to the mean evolution of bubble clouds properties, the dotted line corresponds to the maximal evolution of an 'extreme' bubble cloud property.

- For the configuration 3 with current and motions (Figure 16), the instant $t=0$ corresponds to the peak of the pitch (highest position of the bow). During the 3 minutes of acquisition, we detect 10 bubble clouds around the PP, 5 around the IB and 8 around the TB. The bubble clouds mean area is slightly lower than $0.025 A_{0}$ for the classic and inverted bows. The mean depth reaches a value of $0.27 Z_{0}$ for the classic bow of PP, $0.24 Z_{0}$ for the IB and about $0.22 Z_{0}$ for the TB. We can note that we have probably the same behaviour in terms of cloud area and depth compared to configuration 1 . The reason is that the significant clouds are mostly due to vortex shedding clouds closing to the first configuration. However, the mean vertical velocities vary between $-0.1 \mathrm{U}$ and $0.26 \mathrm{U}$. These values are higher to configuration 1 , probably due to the model motions but not enough to be really 
problematic for acoustic surveys.
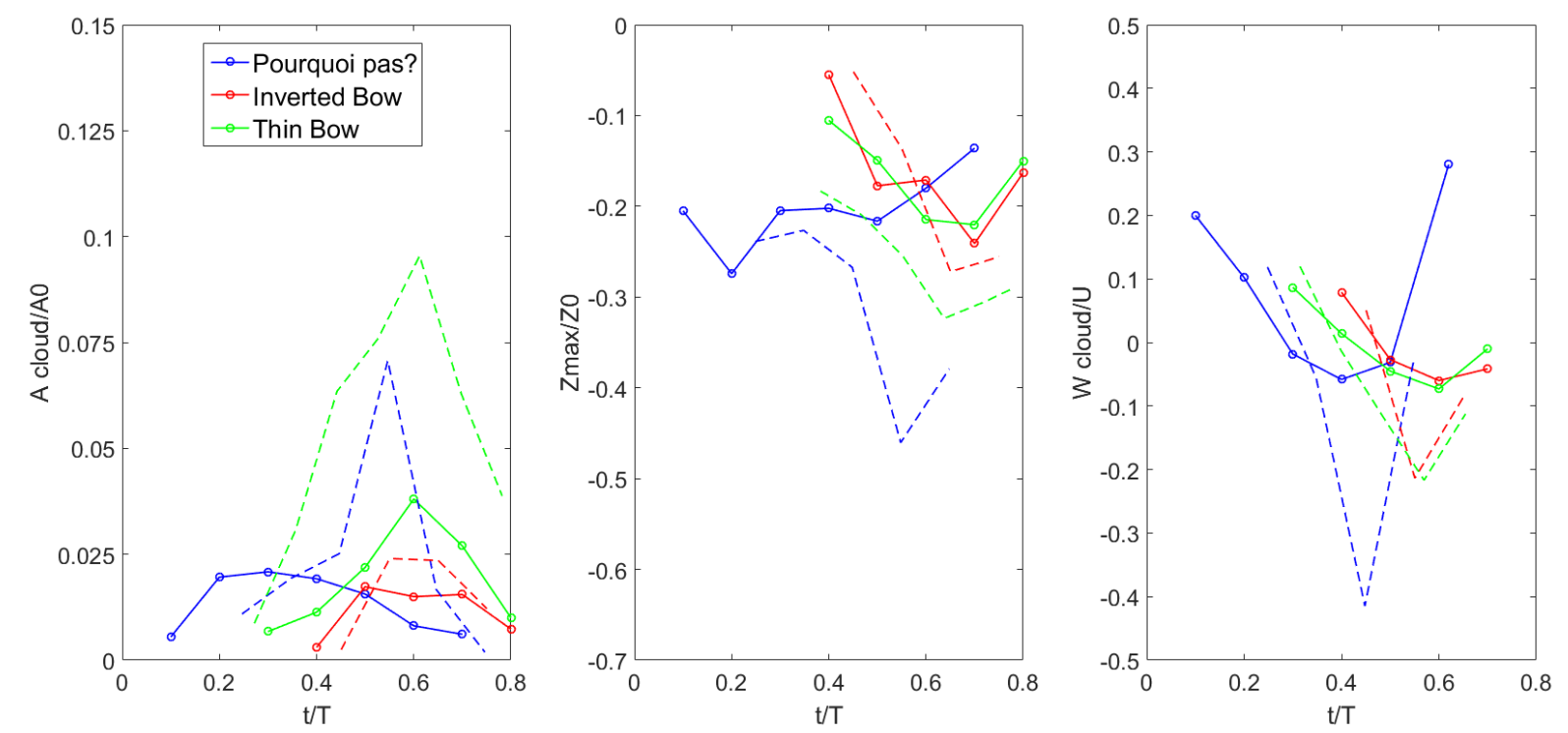

Figure 16: The bubble clouds properties (area, maximal depth and vertical velocity) in configuration 3 (current and motions) around the three ship models. Each line corresponds to the mean evolution of bubble clouds properties, the dotted line corresponds to the maximal evolution of an 'extreme' bubble cloud property.

- For the configuration 4 with current, waves and motions (Figure 17), the instant $\mathrm{t}=0$ corresponds to the peak of the incoming wave at the bow. During the 3 minutes of acquisition, we detect 39 bubble clouds around the PP, 17 around the IB and 27 around the TB. The combination between waves and ship motions allows to generate siginificant clouds with a mean area of about $0.05 A_{0}$ for the $\mathrm{TB}, 0.04 A_{0}$ for the classic bow and $0.055 A_{0}$ for the IB. The mean depth reaches a value between $0.4 Z_{0}$ and $0.45 Z_{0}$ and the mean vertical velocities vary between $-0.2 \mathrm{U}$ and $0.33 \mathrm{U}$ for the three ship models. In this configuration, bubble clouds have the highest value of area, depth and vertical velocity. Compared to configuration 2, there is no real difference between the three kinds of hull. This is due to the combination between waves and ship motions, with the assumption that all the kinds of ship have the same behaviour at sea. 

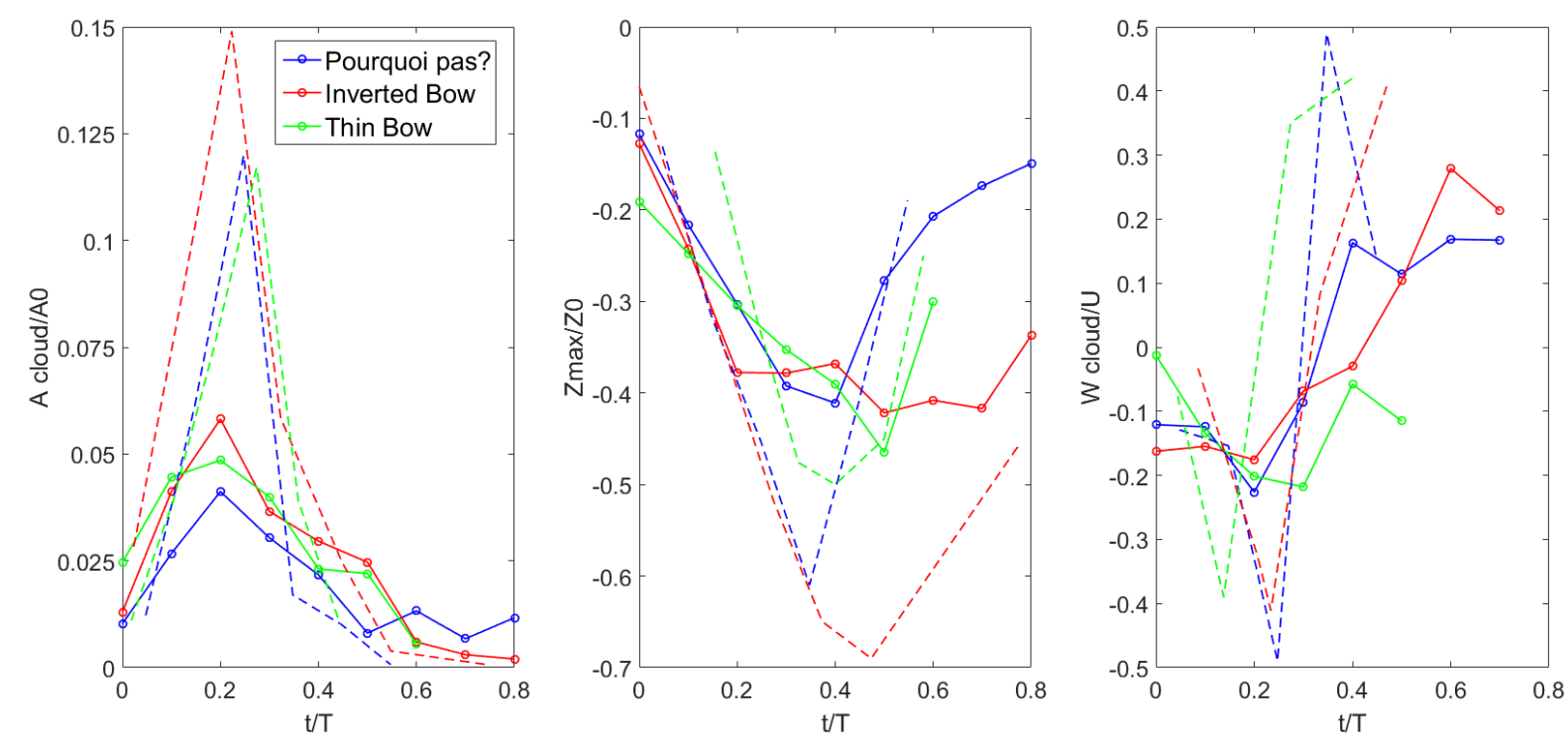

Figure 17: The bubble clouds properties (area, maximal depth and vertical velocity) in configuration 4 (current, waves and motions) around the three ship models. Each line corresponds to the mean evolution of bubble clouds properties, the dotted line corresponds to the maximal evolution of an 'extreme' bubble cloud property.

The bubble clouds properties around the $1 / 30$ scale ship models are converted to full scale in table 4 using the Froude similarity (x $30^{2}$ for the bubble cloud area A, x 30 for the bubble cloud depth Z, $\mathrm{x} \sqrt{30}$ for the bubble cloud vertical velocity W). From all these observations in the four configurations, we can note that the interaction between the wave and the bow has an important role acting on the bubble cloud dynamics especially around the classic bow of the PP. The values of each variable (area, maximum depth and vertical velocity) are also very high when we have a combination of waves and ship motions even if the ship motions seem to counteract the hull geometry effects. In the two cases, whether with only waves or with waves and ship motions, acoustic surveys can be disrupted around the classic bow of the PP especially due to a highest occurrence frequency of bubble clouds in these two configurations (see section 3.1). This conclusion has to be balanced bearing in mind that the behaviour at sea for equivalent ships would not be the same. For the TB, the pitch motion should be higher while for the IB it should be lower with a higher penetration in waves.

In the following, two configurations around the classic bow of the PP and the IB will be analyzed, based on PIV measurements and post-processing POD application. The identification of the flow motion that is responsible for the air entrainment will be investigated. 


\begin{tabular}{|l|c|c|c|c|c|c|c|c|c|}
\hline & \multicolumn{3}{|c|}{ Pourquoi pas? } & \multicolumn{3}{c|}{ Inverted Bow } & \multicolumn{3}{c|}{ Thin Bow } \\
\hline & $A\left(m^{2}\right)$ & $Z(m)$ & $W(m / s)$ & $A\left(m^{2}\right)$ & $Z(m)$ & $W(m / s)$ & $A\left(m^{2}\right)$ & $Z(m)$ & $W(m / s)$ \\
\hline configuration 1 & 2.03 & 1.09 & \pm 0.74 & 2.03 & 0.98 & \pm 0.41 & 3.42 & 1.46 & \pm 0.62 \\
\hline configuration 2 & 5.08 & 2.62 & \pm 1.35 & 1.32 & 1.36 & \pm 0.82 & 2.18 & 2.04 & \pm 0.82 \\
\hline configuration 3 & 2.54 & 1.47 & \pm 0.82 & 2.54 & 1.31 & \pm 0.41 & 4.78 & 1.60 & \pm 0.41 \\
\hline configuration 4 & 4.06 & 2.29 & \pm 1.10 & 5.59 & 2.18 & \pm 1.02 & 6.84 & 3.28 & \pm 0.82 \\
\hline
\end{tabular}

Table 4: The mean values of the bubble cloud properties (area A, depth Z, vertical velocity W) converted from model to full scale for the four configurations around the three models.

\section{Flow analysis}

Two parts are presented in this section. The first part presents the PIV parameters and the phase average method used to determine velocity fields around the classic bow of PP and the IB. The second part is devoted to the flow analysis in the symmetrical plane of the two ship models. In this area, we have more details about the beginning of the breaking wave process (see figure 18). A POD analaysis is proposed allowing to filter the noise velocity measurements in order to identify the flow motion that is significantly responsible of the air entrainment. The configuration 2 (with current and waves) and the configuration 4 (with current, waves and ship motions) are adressed.

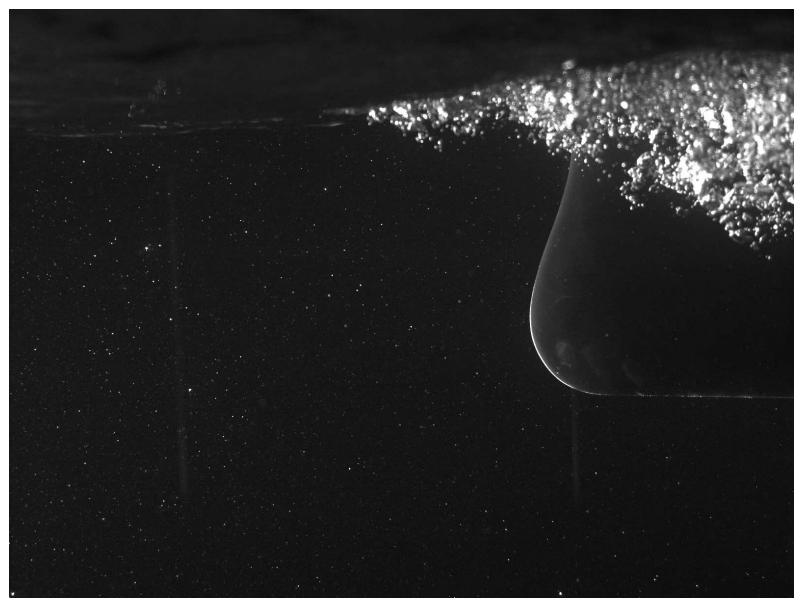

Figure 18: Illustration of images taken in the symmetrical plane $y=0 \mathrm{~mm}$ where a bubble cloud is generated around the classic bow of PP.

\subsection{PIV parameters and phase average method}

PIV measurements have been taken during 180 s with a frequency of $8.5 \mathrm{~Hz}$ to obtain exactly 10 velocity fields per wave period. The main parameters of the PIV databases are recalled in table 5. Indeed, PIV investigation in such a flow configuration can be problematic because of the presence of the oscillating wave surface, of bubbles and of many reflections. In order to overcome these effects, we first detect automatically the 
unsteady free water-air interface oscillations [14]. Figure 19 shows the water-air interface detection (dotted blue line) for the PIV plane $\mathrm{y}=0 \mathrm{~mm}$. The red line correspond to the contours of the hull.

\begin{tabular}{|c|c|}
\hline Parameters & Configurations \\
\hline $\begin{array}{c}\text { Physical dimension (PIV plane) } \\
\text { Number of grid points }\end{array}$ & $\begin{array}{c}528.1 \times 396.1 \mathrm{~mm}^{2} \\
\left(n_{x}, n_{z}\right)=(66,49)\end{array}$ \\
\hline Sampling frequency $f_{e}(\mathrm{~Hz})$ & 8.5 \\
\hline Number of PIV snapshots & 1530 \\
\hline
\end{tabular}

Table 5: Main parameters of the PIV databases under consideration for the symmetrical plane $\mathrm{y}=0 \mathrm{~mm}$.

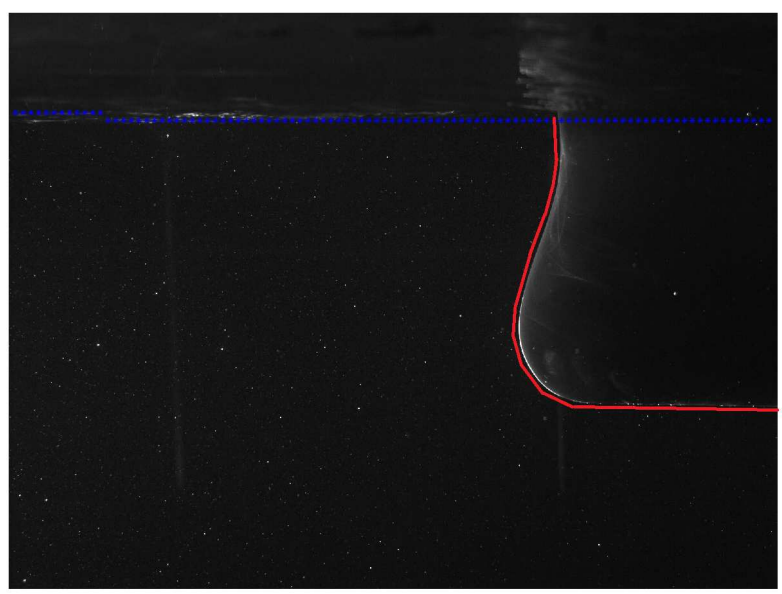

Figure 19: Illustration of the water-air interface detection (dotted blue line) applied on consecutive raw PIV images. The red line corresponds to the hull contours in the symmetrical plane $\mathrm{y}=0 \mathrm{~mm}$.

The results in the following are presented in term of phase average for which the vertical velocity component is investigated. The phase average is calculated by readjusting the phase from the wave signal measured by the resistive probe. For each period, the image corresponding to the wave peak at the bow is detected and added to compute the phase average denoted 5 (see figure 20). For each phase from 1 to 9 , we compute the average of the water-air interface position for all images corresponding to the associated phase and we apply the resulted free surface mask on the average of the velocity fields for all images in this same phase. We know that the water-air interface is not always in the same location in each phase, but we assume that the variation of this interface is negligible (a few $\mathrm{mm}$ in a PIV plane of $400 \mathrm{~mm}$ width). Recalling the sampling frequency of measurements is $10 \times f_{0}$, then these 9 consecutive snapshots correspond approximately to a period of $1 / f_{0}$. Each image from 1 to 9 in the following figures corresponds to the average of the velocity fields in all periods and corresponds to the phases provided in figure 20. For reasons of clarity, the phase 10 is not shown but it has been taken into account during the analysis. 


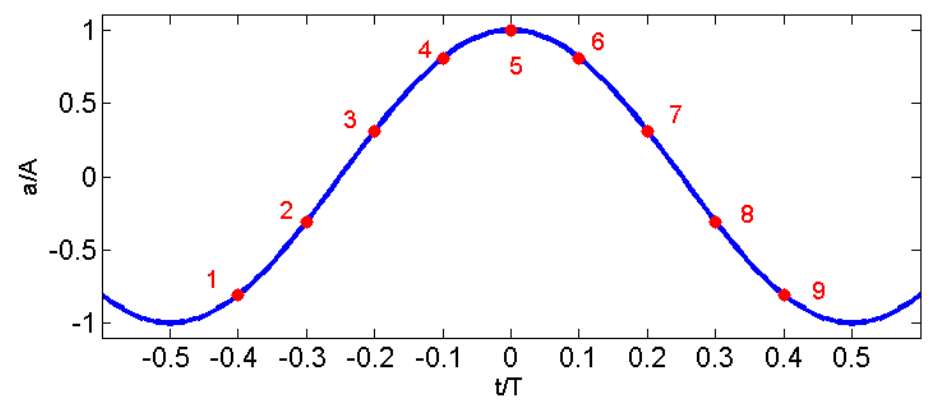

Figure 20: Time decomposition along a regular wave signal for phase average calculation.

\subsection{Flow motion vs Bubble generation}

In order to investigate the flow in the area where breaking waves take place, we do measurements in the symmetrical plane $\mathrm{y}=0 \mathrm{~mm}$. In this plane, few bubbles are observed. Therefore, a POD analysis can be used.

After applying a dynamic mask to each instantaneous PIV velocity vector field, a POD analysis is carried out in order to reconstruct the phase average velocity field from each available PIV velocity field. The POD analysis proposed here is only a denoising tool that allows to smooth the velocity fields. Following previous works ([11], [2]), we propose to implement the phase average operator based on POD application. Briefly, as POD is based on a energetic optimization problem, an approximation of a phase average flow field is obtained thanks to a linear combination of the first POD modes ([11], [2]). In a certain sense, such POD application can be viewed as a denoising tool that allows the determination of phase average velocities fields.

POD is performed from the $N t=1530$ instantaneous longitudinal and transverse components of the velocity field accessed on a regular grid mesh of $(n x \times n z=66 \times 49$ points. The energetic content of the first three POD modes represents about $82 \%$ of the total energy. The first POD spatial eigenfunctions as well as the first POD temporal coefficients are analyzed similarly than in previous works ([11], [2]). Then it is demonstrated that the projection of each instantaneous velocity field onto the first three POD modes leads to obtain an instantaneous dominant flow field that corresponds to the phase average velocity field.

Some instantaneous consecutive velocity fields with streamlines deduced from the phase average flow field are plotted in figure 21 (configuration 2) and figure 22 (configuration 4). It is observed that the motion of the hull (see figure 22) greatly affects the phase average flow, especially above the bulb of the hull. In this zone, the ascending velocities are high and reach a maximal value of $0.18 \mathrm{~m} / \mathrm{s}$ for the configuration 4 and a value of $0.1 \mathrm{~m} / \mathrm{s}$ for the configuration 2 . Indeed, when comparing the two representations, the presence of a vertical mean flow is quite pronounced near the hull in configuration 
4 in comparison with the results of configuration 2 , for which the vertical component is only due to the bow wave. Therefore, the motion of the hull drives the fluid entrainment towards the top.
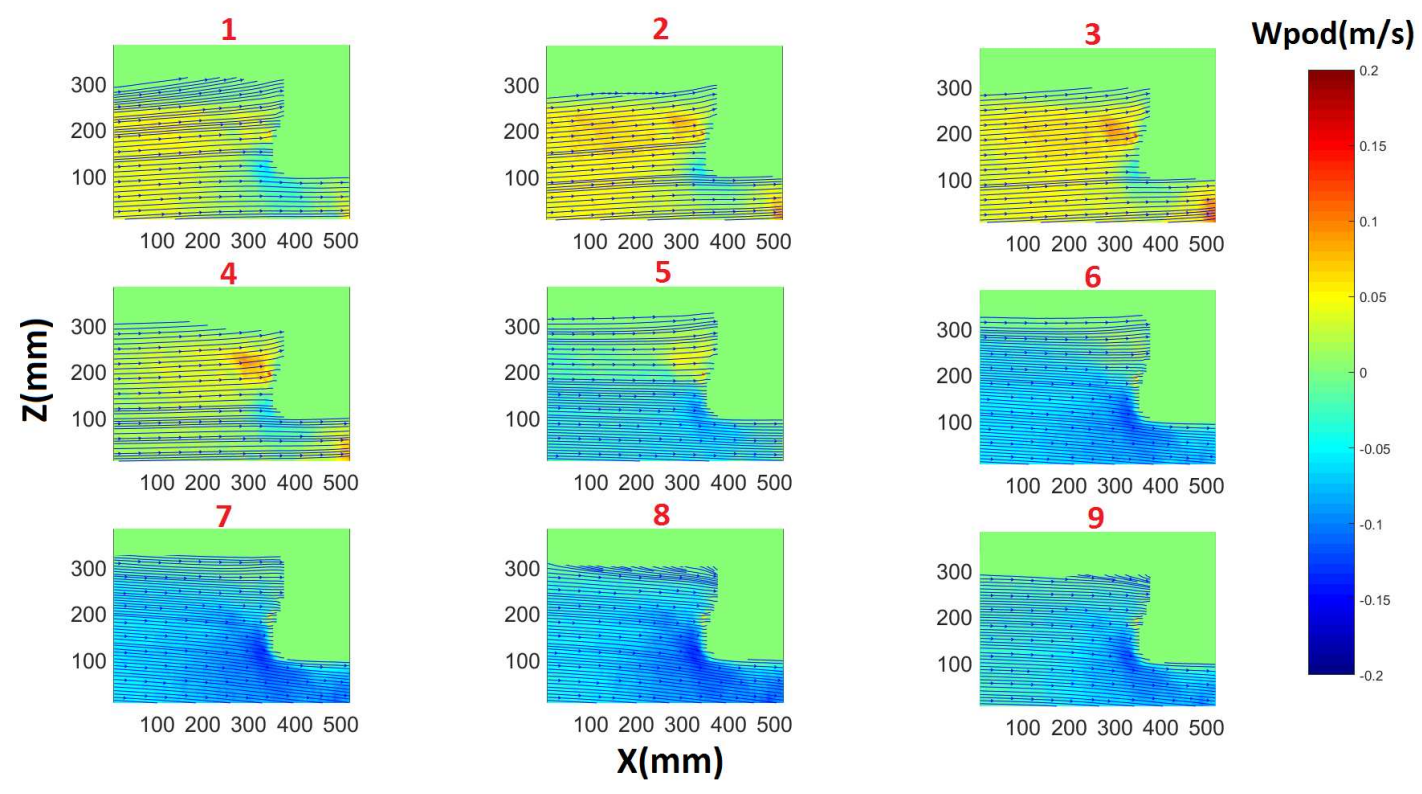

Figure 21: Representation of nine consecutive velocity fields with streamlines deduced from the projection of velocity field onto the first three POD modes for configuration 2 around the PP. The colormap corresponds to the vertical velocity component Wpod.
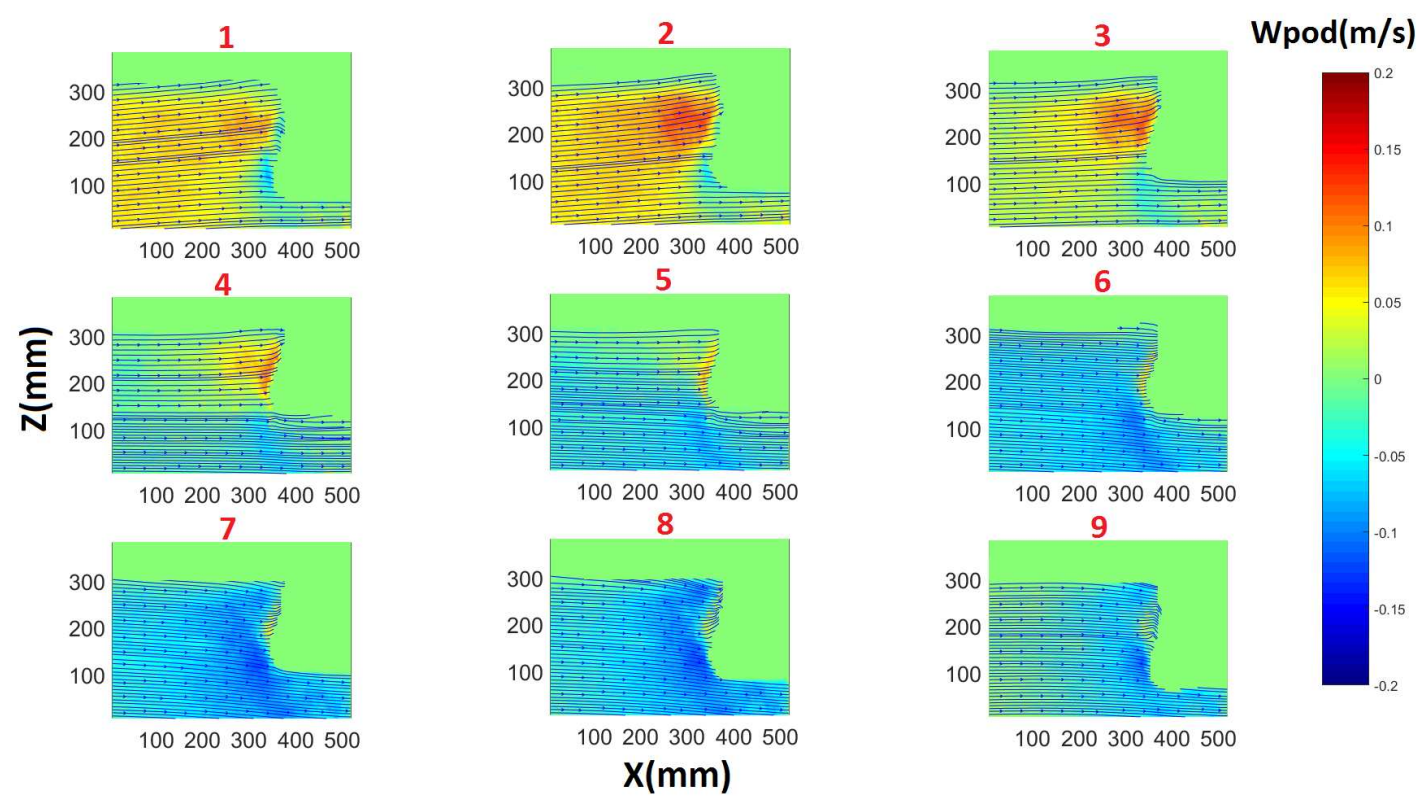

Figure 22: Representation of nine consecutive velocity fields with streamlines deduced from the projection of velocity field onto the first three POD modes for configuration 4 around the PP. The colormap corresponds to the vertical velocity component Wpod. 
The same POD analysis has been performed around the IB during the same experimental time duration of $180 \mathrm{~s}$. Figure 23 and figure 24 present some instantaneous consecutive velocity fields with streamlines deduced from the phase average flow field for the configuration 2 and 4 respectively around the IB. In this case, contrary to the case of $\mathrm{PP}$, there is no a separation zone with a positive vertical velocity above the bulb of the bow and a negative vertical velocity below it. The flow is almost uniform around the IB for the configuration 2, except the area under the bow from phases 6 to 9 . The ascending vertical velocities reach a maximal value of $0.08 \mathrm{~m} / \mathrm{s}$. In this area, the plunging vertical velocities are high in contact with the bow. It is due to the geometry of the IB that allows to generate plunging streamlines (see figure 23 and figure 24), more pronounced than around the classic bow of PP. Regarding the configuration 4, the flow is always uniform during phases 1 to 5 with a little increase of the ascending velocities in the contact zone between the wave and the model in phases 3 and 4 . The ascending vertical velocities reach a maximal value of $0.12 \mathrm{~m} / \mathrm{s}$.
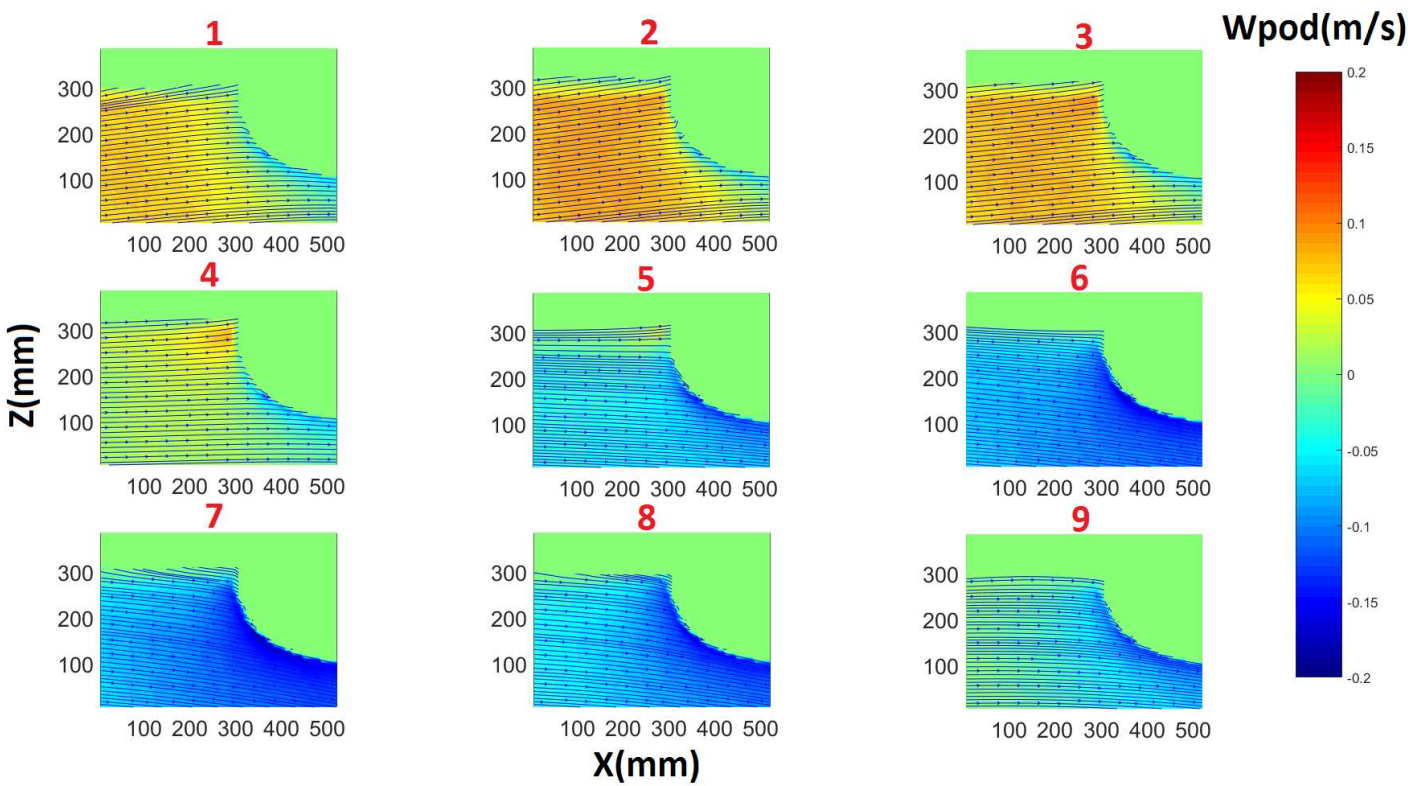

Figure 23: Representation of nine consecutive velocity fields with streamlines deduced from the projection of velocity field onto the first three POD modes for configuration 2 around the IB. The colormap corresponds to the vertical velocity component Wpod.

To confirm these observations, figure 26 and 27 present the evolution of the difference of the transverse velocity components $d \omega=w_{2}-w_{1}$ at two local points around the classic bow of PP and the IB for the configurations 2 and 4 . The vertical velocity at the local points $X_{1}$ and $X_{2}$ correspond respectively to $w_{1}$ and $w_{2}$. The two points are located near the bow and far away from the bow (see figure 25). For the two ship models, the distance between the bow and $X_{2}$ is $d X_{2}=50 \mathrm{~mm}$, the distance between the bottom of the model and the point $X_{1}$ or $X_{2}$ is $\mathrm{dZ}=150 \mathrm{~mm}$, and the distance between $X_{1}$ and $X_{2}$ is $d X_{1}=200 \mathrm{~mm}$. 
1

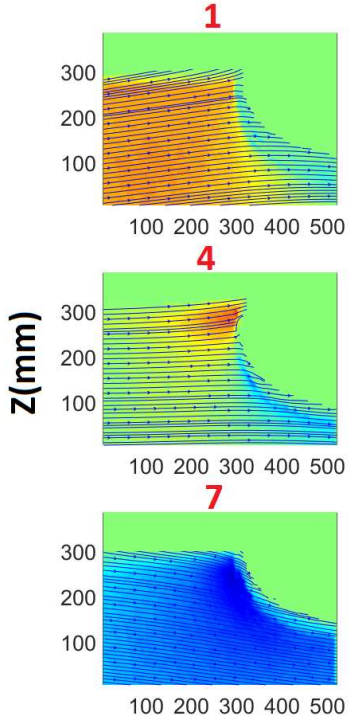

2

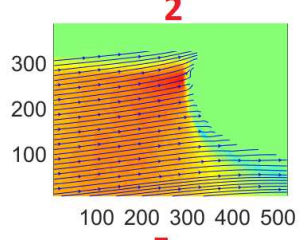

5

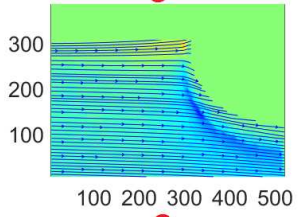

8

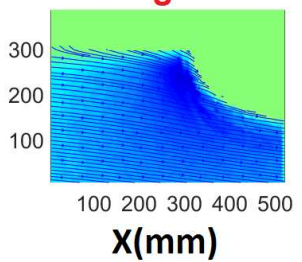

3

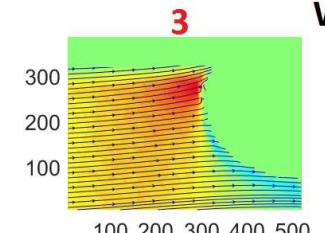

6

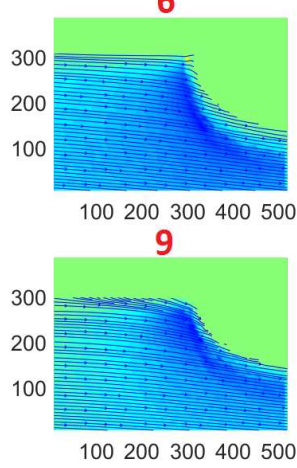

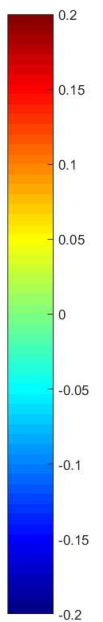

Figure 24: Representation of nine consecutive velocity fields with streamlines deduced from the projection of velocity field onto the first three POD modes for configuration 4 around the IB. The colormap corresponds to the vertical velocity component Wpod.
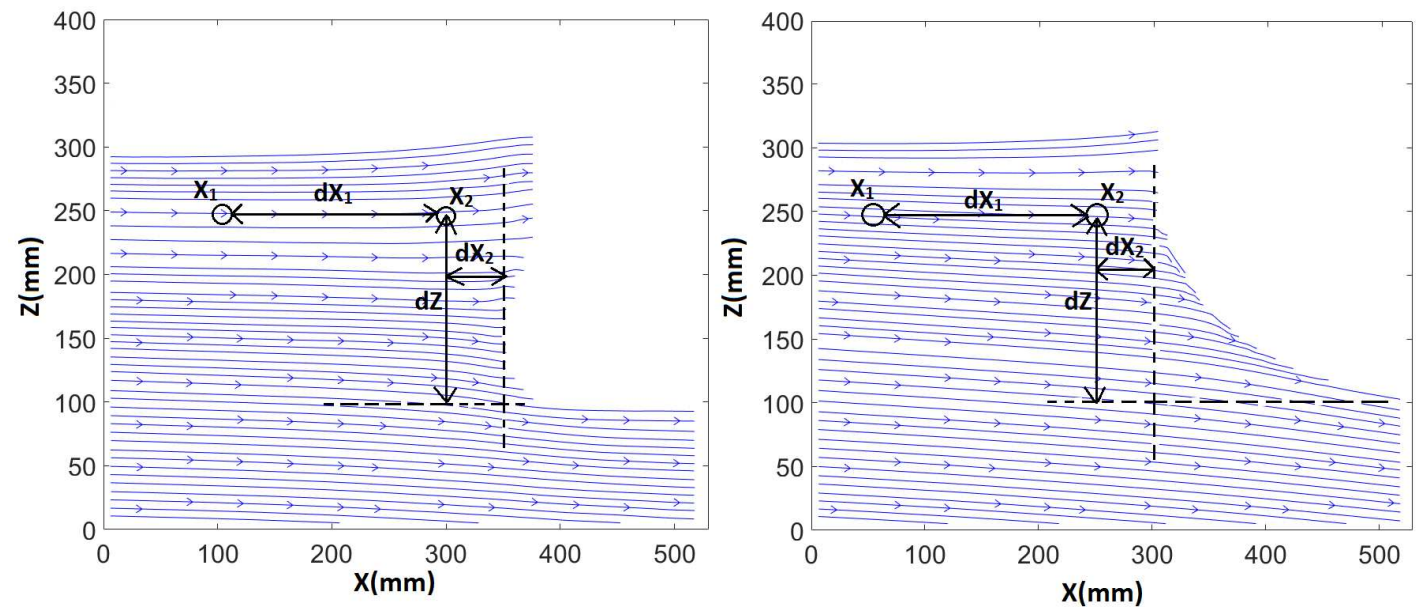

Figure 25: The locations of the two points $X_{1}$ and $X_{2}$ around the PP (left) and the IB (right).

We can see that the variation of $d \omega$ is higher around the classic bow of the PP especially for the configuration 4 . In this case, $\left(d \omega_{\text {config } 4}\right)_{P P}$ oscillates between $d \omega_{\text {mean }} \pm$ $d \omega_{R M S}=0.02 \pm 0.06 \mathrm{~m} / \mathrm{s} . \quad d \omega_{\text {mean }}=\frac{1}{N} \sum_{n=1}^{N}\left(d \omega_{m}\right)_{n}$ and $d \omega_{R M S}=\sqrt{\frac{1}{N} \sum_{n=1}^{N}\left|\left(d \omega_{m}\right)_{n}\right|^{2}}$ correspond respectively to the mean average and the Root-Mean-Square of $d \omega$ with $N$ $=1530$ the number of snapshots. For the configuration 2 around the classic bow of $\mathrm{PP},\left(d \omega_{\text {config } 2}\right)_{P P}$ oscillates between $0.02 \pm 0.03 \mathrm{~m} / \mathrm{s}$. This difference is almost negligible around the IB. It oscillates between $\left(d \omega_{\text {config } 2}\right)_{I B}=0.01 \pm 0.01 \mathrm{~m} / \mathrm{s}$ for the configuration 2 and between $\left(d \omega_{\text {config } 4}\right)_{I B}=-0.02 \pm 0.03 \mathrm{~m} / \mathrm{s}$ for the configuration 4.

The different values of $d \omega_{\text {mean }}, d \omega_{R M S}$ and the occurrence frequency of bubble clouds 


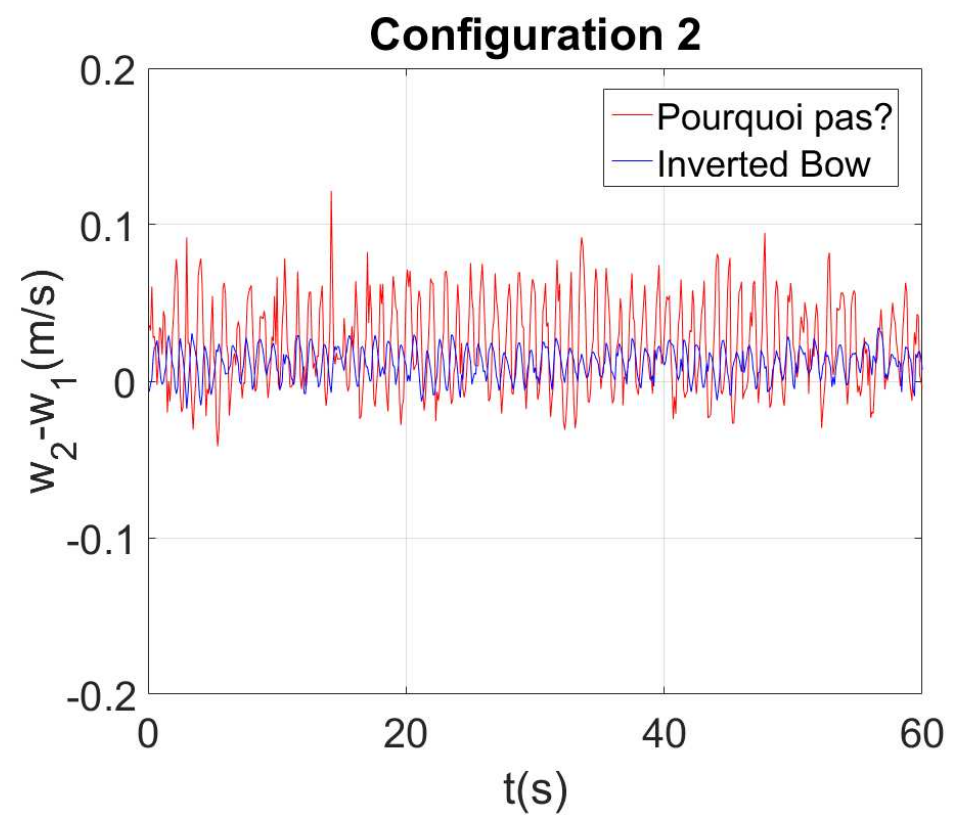

Figure 26: Evolution of the difference of the vertical velocity $d \omega=w_{2}-w_{1}$ at the point $X_{1}$ and $X_{2}$ in the configuration 2 with waves around the classic bow of PP and the IB.

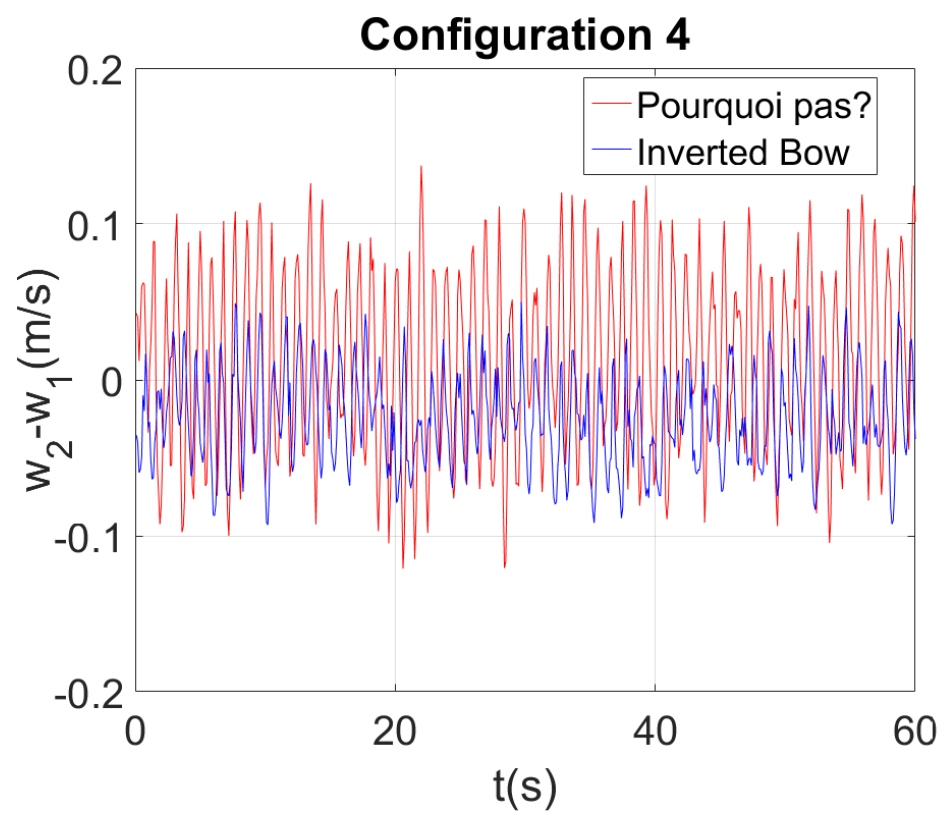

Figure 27: Evolution of the difference of the vertical velocity $d \omega=w_{2}-w_{1}$ at the point $X_{1}$ and $X_{2}$ in the configuration 4 with waves around the classic bow of PP and the IB.

$f / f_{0}$ for the two configurations around the two ship models are summarized in table 6 .

Two comparisons can be done here: i) the comparison between the configurations 2 and 4 for the same ship model and ii) the comparison between the two ship models for the same configuration. For i), we can see that the values are always more important for the configuration 4 comparing to the configuration 2 around the classic bow of the PP, as around the IB. For ii), we note that the values around the classic bow of the PP are almost two times higher than the values around the IB for the configuration 2, as for the 
configuration 4. The two comparisons indicate that the combination between waves and ship motions is the origin not only to high occurrence frequency of bubble clouds as shown in 3.1, but also to high variations of the phase average velocity fields near the bow. All these observations confirm that the hull motions lead to a great flow entrainment towards the top around the classic bow of PP. Then as a function of the vertical flow intensity, the resulted interaction with the free surface generates high amplitude bow wave.

\begin{tabular}{|l|l|c|c|c|}
\hline & & $f / f_{0}$ & $d \omega_{\text {mean }}(\mathrm{m} / \mathrm{s})$ & $d \omega_{R M S}(\mathrm{~m} / \mathrm{s})$ \\
\hline \multirow{2}{*}{ Pourquoi pas? } & config 2 & 0.48 & 0.02 & 0.03 \\
& config 4 & 0.57 & 0.02 & 0.06 \\
\hline \multirow{2}{*}{ Inverted Bow } & config 2 & 0.18 & 0.01 & 0.01 \\
& config 4 & 0.37 & -0.02 & 0.03 \\
\hline
\end{tabular}

Table 6: The different values of the mean average $d \omega_{\text {mean }}$ and the Root-Mean-Square $d \omega_{R M S}$ of the difference of the vertical velocity component at two local points for the configuration 2 and 4 around the classic bow of PP and IB. The occurrence frequency of bubble clouds is $f / f_{0}$.

As conclusion, we note that the flairing of the bow of the PP is the origin of a high ascending velocity concentrated above the bulb of the bow. The intensity of the flow is high in this region. Therefore, a high interaction between the incoming waves and the bow generates more intense breaking waves and consequently more bubbles. On the other hand, the geometry of the IB is the origin of a small interaction between the incoming waves and the bow that allows to generate less intense breaking waves and consequently fewer bubbles.

\section{Conclusions and future work}

An experimental study of the bubble sweep-down phenomenon around three ship models have been carried out to assess the effects of the bow geometry on bubble generation. Through the experimental configurations, two different air entrainment mechanisms have been detected around the three ship bow models. The first one corresponds to the breaking of the bow wave and the second is the air entrainment by vortex shedding cloud.

The occurrence frequency of bubble clouds for four configurations and for the three models have been computed. The results showed that in the configuration with waves, the generation of bubbles is bigger for the classic bow of the PP compared to the inverted and thin bows. It is directly due to the flaring of the bow of the PP which induces more intense breaking waves and so the generation of more bubble clouds.

An image processing method via a gray-scale analysis has been used to detect and follow the evolution of the bubble clouds. Bubble cloud properties in terms of area, maximum depth and vertical velocity have been computed and then compared for the three models. The results showed that the number of significant bubble clouds around 
the inverted and thin bow is low compared to the classic bow of the PP. Bubble clouds

[1] P. Bonmarin. Geometric properties of deep-water breaking waves. J. Fluid Mech., 209:405-433, 1989.

PIV measurements followed by a mathematical post-treatment tool (POD analysis) have been performed to extract the phase average velocity flow field in the symmetrical plane of the classic bow of the PP and the IB. It is observed that the ship motions combined with waves increase not only the occurrence frequency of bubble clouds, but also the amplitude of the ascending vertical velocity component above the bulb of the classic bow of the PP than elsewhere in the PIV plane. Furthermore, the hull shape has a significant influence onto the hydrodynamic properties of the flow and on the interaction between the incoming waves and the bow. Therefore, the bow geometry is a very important parameter acting on the intensity of the breaking waves and consequently on the bubble generation.

Even if scale effects are significant and the results in term of bubble dynamics are not the same in the flume tank and at real scale, this study allows the quantification of the bubble sweep-down phenomenon around different bow geometries under waves and current in order to compare the performance of each bow geometry face to this problem. The overall results provide an interesting experimental database for the hull ship designers and could be used for the validation of future numerical models, knowing that these results enable the identification of the areas where flow and body interaction is pronounced and then the location of the bubble cloud generation and propagation.

Techniques for measuring free surface deformation $[16,15]$ in the contact zone with the bow shape is our interest in the near future to understand the link between free surface elevation and bubble generation better. The density of bubbles, an important parameter for acoustic perturbation considerations, will be investigated by the use of an optical probe system located around the ship bow where the bubble sweep-down phenonmenon takes place. The knowledge of the 3D behaviour of the bubble clouds should be also studied.

\section{Acknowledgements}

The authors would like to thank Ifremer and the Hauts-de-France Regional Council for the financial funding of the $\mathrm{PhD}$ project. We are also grateful to Thomas Bacchetti and Jean-Valery Facq for their assistance in the design and set-up of these experiments.

\section{References}


[2] E. Bouhoubeiny, P. Druault, and G. Germain. Phase-averaged mean properties of turbulent flow developing around a fluttering sheet of net. Ocean Engineering, 82:160-168, 2014.

[3] P. M. Carrica, D. Drew, F. Bonetto, and R.T. Jr Lahey. A polydisperse model for bubbly two-phase flow around a surface ship. International Journal of Multiphase Flow, 25:257-305, 1999.

[4] A.M. Castro and P.M. Carrica. Eulerian polydispersed modeling of bubbly flows around ships with application to athena R/V. Int. Shipbuilding Progr., 60:403-433, 2013.

[5] G.B. Deane and M.D. Stockes. Scale dependence of bubble creation mechanisms in breaking waves. Nature, 418:839-844, 2002.

[6] S. Delacroix. Caractérisation de la génération et de la propagation de bulles autour de la carène des navires scientifiques. PhD thesis, Université de Bretagne Occidentale, 2014.

[7] S. Delacroix, G. Germain, L. Berger, and J-Y. Billard. Bubble sweep-down occurrence characterization on research vessels. Ocean Engineering, 111:34-42, 2016.

[8] S. Delacroix, G. Germain, B. Gaurier, and J-Y. Billard. Experimental study of bubble sweep-down in wave and circulating tank. bubble clouds characterization. Ocean Engineering, 120:88-99, 2016.

[9] S. Delacroix, G. Germain, B. Gaurier, and J-Y. Billard. Experimental study of bubble sweep-down in wave and circulating tank. experimental set-up and observed phenomena. Ocean Engineering, 120:78-87, 2016.

[10] G. Delhommeau, M. Guilbaud, L. David, C. Yang, and F. Noblesse. Boundary between unsteady and overturning ship bow wave regimes. Flow Turbulence Combust, 620:167-175, 2009.

[11] P. Druault, E. Bouhoubeiny, and G. Germain. Pod investigation of the unsteady turbulent boundary layer developing over porous moving flexible fishing net structure. Exp. Fluids, 53:277-292, 2012.

[12] J. H. Duncan. An experimental investigation of breaking waves produced by a towed hydrofoil. Proc. R. Soc. A, 377:331-348, 1981.

[13] J. H. Duncan. The breaking and non-breaking wave resistance of a two-dimensional hydrofoil. Journal of Fluid Mechanics, 126:507-520, 1983. 
[14] D. Dussol, P. Druault, B. Mallat, S. Delacroix, and G. Germain. Automatic dynamic mask extraction for piv images containing an unsteady interface, bubbles, and a moving structure. C.R. Mecanique, 344:464-478, 2016.

[15] G. Gomit, L. Chatellier, D. Calluaud, and L. David. Free surface measurements by stereo-refraction. Exp. Fluids, 54:1540, 2013.

540 [16] L. Gui, H. Yoon, and F. Stern. Techniques for measuring bulge-scar pattern of free surface deformation and related velocity distribution in shallow water flow over a bump. Exp. Fluids, 55:1-15, 2014.

[17] E. Lamarre and W. K. Melville. Void-fraction measurements and sound-speed fields in bubble plumes generated by breaking waves. J. Acoust. Soc. Am., 95:1317-1328, 1994.

[18] J. Ma, A. A. Oberai, M. C. Hyman, D. A. Drew, and R.T. Jr Lahey. Two-fluid modeling of bubbly flows around surface ships using a phenomenological subgrid air entrainment model. Computers \&f Fluids, 52:50-57, 2011.

[19] F.J. Moraga, P.M. Carrica, D.A. Drew, and R.T. Jr Lahey. A sub-grid air entrainment model for breaking bow waves and naval surface ships. Computer \& Fluids, 37:281-298, 2008.

[20] F. Noblesse, G. Delhommeau, M. Guilbaud, D. Hendrix, and C. Yang. Simple analytical relations for ship bow waves. Journal of Fluid Mechanics, 600:105-132, 2008.

[21] F. Noblesse, G. Delhommeau, M. Guilbaud, H. Liu, D. Wan, and C. Yang. Ship bow waves. Journal of Hydrodynamics, 25:491-501, 2013.

[22] M. Tavakolinejad. Air bubble entrainment by breaking bow waves simulated by a 2D + T technique. PhD thesis, University of Maryland, 2010.

[23] V.M. Trenkel, L. Berger, S. Bouguignon, M. Doray, R. Fablet, J. Mass, V. Mazauric, C. Poncelet, L. Quemener, C. Scalabrin, and H. Villalobos. Overview of recent progress in fisheries acoustics made by ifremer with examples from the bay of biscay. Aquat. Living Resour., 22:433-445, 2009.

[24] T.A. Waniewski, C.E. Brennen, and F. Raichlen. Measurements of air entrainment by bow waves. J. Fluids Eng, 123:57-63, 2001. 